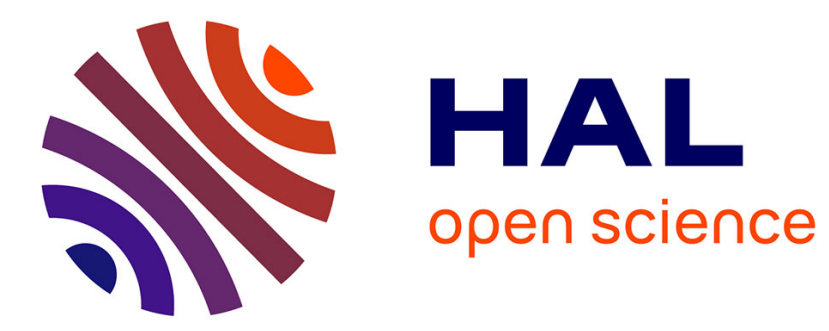

\title{
Where neuroscience and dynamic system theory meet autonomous robotics: A contracting basal ganglia model for action selection
}

\author{
Benoît Girard, N Tabareau, Q C Pham, A. Berthoz, J.-J C Slotine
}

\section{- To cite this version:}

Benoît Girard, N Tabareau, Q C Pham, A. Berthoz, J.-J C Slotine. Where neuroscience and dynamic system theory meet autonomous robotics: A contracting basal ganglia model for action selection. Neural Networks, 2008, 21 (4), pp.628- 641. 10.1016/j.neunet.2008.03.009 hal-01524676

\section{HAL Id: hal-01524676 \\ https://hal.science/hal-01524676}

Submitted on 18 May 2017

HAL is a multi-disciplinary open access archive for the deposit and dissemination of scientific research documents, whether they are published or not. The documents may come from teaching and research institutions in France or abroad, or from public or private research centers.
L'archive ouverte pluridisciplinaire HAL, est destinée au dépôt et à la diffusion de documents scientifiques de niveau recherche, publiés ou non, émanant des établissements d'enseignement et de recherche français ou étrangers, des laboratoires publics ou privés. 


\title{
Where neuroscience and dynamic system theory meet autonomous robotics: a contracting basal ganglia model for action selection
}

\author{
B. Girard ${ }^{a, b, *}$ N. Tabareau ${ }^{a, b}$ Q.C. Pham ${ }^{a, b}$ A. Berthoz ${ }^{a, b}$ \\ J.-J. Slotine ${ }^{c}$ \\ ${ }^{a}$ Laboratoire de Physiologie de la Perception et de l'Action, Collège de France, 11 place \\ Marcelin Berthelot, 75231 Paris Cedex 05, France. \\ ${ }^{\mathrm{b}}$ UMR 7152, CNRS, 11 place Marcelin Berthelot, 75231 Paris Cedex 05, France. \\ ${ }^{\mathrm{c}}$ Nonlinear Systems Laboratory, Massachusetts Institute of Technology, Cambridge, \\ Massachusetts, 02139, USA.
}

\begin{abstract}
Action selection, the problem of choosing what to do next, is central to any autonomous agent architecture. We use here a multidisciplinary approach at the convergence of neuroscience, dynamical systems theory and autonomous robotics, in order to propose an efficient action selection mechanism based on a new model of the basal ganglia. We first describe new developments of contraction theory regarding locally projected dynamical systems. We exploit these results to design a stable computational model of the cortico-basothalamo-cortical loops. Based on recent anatomical data, we include usually neglected neural projections, which participate in performing accurate selection. Finally, the efficiency of this model as an autonomous robot action selection mechanism is assessed in a standard survival task. The model exhibits valuable dithering avoidance and energy-saving proper-
\end{abstract}


ties, when compared with a simple if-then-else decision rule.

Key words: action selection, basal ganglia, computational model, autonomous robotics, contraction analysis

\section{Contents}

1 Introduction

2 Nonlinear contraction analysis for rate coding neural networks

2.1 Contraction theory

2.2 Neural networks and locally projected dynamical systems

2.3 Contraction analysis of locally projected dynamical system on regular $n$-cubes

2.4 Combination of contracting systems

3 Model description

$4 \quad$ Disembodied model results

4.1 Contraction analysis of the model

4.2 Basic selection test

4.3 Systematic salience search test

$5 \quad$ Minimal survival task

5.1 Material and methods

* corresponding author. Tel.: +3314427 13 91; fax: +33 1442713 82.
Email address: benoit.girardecollege-de-france.fr(B. Girard). 
6 Discussion

$\begin{array}{lll}6.3 & \text { Autonomous robotics } & 38\end{array}$

$\begin{array}{lll}\text { A If-Then-Else decision rule } & 40\end{array}$

B Robot CBG saliences $\quad 41$

\section{Introduction}

Action selection is the problem of motor resource allocation an autonomous agent is faced with, when attempting to achieve its long-term objectives. These may vary from survival and reproduction to delivering letters to researchers' offices, depending on the nature of the considered agent (animal, robot, etc.). Action selection is a topic of interest in various disciplines, including ethology, artificial intelligence, psychology, neuroscience, autonomous robotics, etc. We address here the question of action selection for an autonomous robot, using a computational model of brain regions involved in action selection, namely the cortico-baso-thalamo-cortical loops. In order to avoid unwanted dynamical behaviors resulting from a highly recurrent network, we use contraction analysis (Lohmiller and Slotine, 1998) to obtain a rigorous proof of its stability. The efficiency of this action selection mechanism (ASM) is assessed using a standard minimal survival task in a robotic simulation. 
[Fig. 1 about here.]

The basal ganglia are a set of interconnected subcortical nuclei common to all vertebrates and involved in numerous processes, from motor functions to cognitive ones (Mink, 1996; Middleton and Strick, 1994). Their role is interpreted as a generic selection circuit, and they have been proposed to form the neural substrate of action selection (Mink, 1996; Krotopov and Etlinger, 1999; Redgrave et al., 1999). The basal ganglia are included in cortico-baso-thalamo-cortical loops (Fig. B.1), five main loops have been identified in primates (Alexander et al., 1986, 1990; Kimura and Graybiel, 1995): one motor, one oculomotor, two prefrontal and one limbic loop. Within each of these loops, the basal ganglia circuitry is organized in interacting channels, among which selection occurs. Depending on the loop considered, this selection may concern, for example, the target of an upcoming saccadic movement, the target of reaching movement or the piece of information to be stored in working memory. The output nuclei of the basal ganglia are inhibitory and tonically active, and thus maintain their targets under sustained inhibition. Selection occurs via disinhibition (Chevalier and Deniau, 1990): the removal of the inhibition exerted by one channel on its specific target circuit allows the activation of that circuit. When considering action selection, the basal ganglia channels are thought to be associated to competing action primitives. Given sensory and motivational inputs, the basal ganglia are thus supposed to arbitrate among these actions and to allow the activation of the winner by disinhibiting the corresponding motor circuits.

The considered network contains a large number of closed loops, from the large cortico-baso-thalamo-cortical loop, to loops formed by the interconnections between nuclei within the basal ganglia and between the thalamus and the cortex. A system with such a structure may exhibit varied dynamical behaviors, some of which should be avoided by an ASM, like reaching a standstill state which doesn't 
depend anymore on the external input. This motivates the use of a theoretical framework to study the dynamics of basal ganglia models. We propose to use contraction analysis (Lohmiller and Slotine, 1998) in order to guide the design of a new model of the basal ganglia whose stability can be formally established. Contraction analysis is a theoretical tool used to study the dynamic behavior of non-linear systems. Contraction properties are preserved through a number of particular combinations, which is useful for a modular design of models.

Numerous computational models of the BG have been proposed in order to investigate the details of the operation of the basal ganglia disinhibition process (Gillies and Arbruthnott, 2000; Gurney et al., 2004b, for recent reviews). Among these, the model proposed by Gurney, Prescott and Redgrave (2001a; 2001b) (henceforth the GPR model) has been successfully tested as an action selection mechanism for autonomous agents (Montes-Gonzalez et al., 2000; Girard et al., 2003, 2005a; Prescott et al., 2006). In particular, it was shown to be able to solve a minimal survival task, and, compared with a simpler winner-takes-all mechanism, displayed dithering avoidance and energy-saving capabilities.

We present here an action selection mechanism based on a contracting computational model of the basal ganglia (or CBG). In order to adapt the contraction theory to the analysis of rate-coding artificial neural networks, we first extend it to locally projected dynamical systems (section 2). Using the resulting neuron model and contraction constraints on the model's parameters, we build a computational model of the basal ganglia including usually neglected neural connections (section 3). We then check the selection properties of the disembodied model and compare them to those of the GPR, so as to emphasize the consequences of using contraction analysis (section 4). We finally test its efficiency in a survival task similar to the one used to evaluate the GPR (Girard et al., 2003), and emphasize its dithering avoidance 
and energy-saving properties by comparing it to a simple if-then-else decision rule (section 5).

Preliminary versions of the basal ganglia computational model were presented in (Girard et al., 2005b, 2006).

\section{Nonlinear contraction analysis for rate coding neural networks}

Basically, a nonlinear time-varying dynamic system is called contracting if initial conditions or temporary disturbances are forgotten exponentially fast, that is, if any perturbed trajectory returns to its nominal behavior with an exponential convergence rate. Contraction is an extension of the well-known stability analysis for linear systems. It has the desirable feature of being preserved through hierarchical and particular feedback combinations. Thus, as we will see below, contraction analysis is an appropriate tool to study stability properties of rate coding neural networks.

In addition, when a system is contracting, it is sufficient to find a particular bounded trajectory to be sure that the system will eventually tend to this trajectory. Thus contraction theory is a convenient way to analyze the dynamic behavior of a system without linearized approximations.

\subsection{Contraction theory}

We summarize the differential formulation of contraction analysis presented in (Lohmiller and Slotine, 1998). Contraction analysis is a way to prove the exponential stability of a nonlinear system by studying the properties of its Jacobian. 
Consider a $n$-dimensional time-varying system of the form:

$$
\dot{\mathbf{x}}(t)=\mathbf{f}(\mathbf{x}(t), t)
$$

where $\mathbf{x} \in \mathbb{R}^{n}$ and $t \in \mathbb{R}_{+}$and $\mathbf{f}$ is a $n \times 1$ non-linear vector function which is assumed in the rest of this paper to be real and smooth, in the sense that all required derivatives exist and are continuous. This equation may also represent the closedloop dynamic of a neural network model of a brain structure. We recall below the main result of contraction analysis (see Lohmiller and Slotine, 1998, for a proof and more details).

Theorem 1 Consider the continuous-time system (1). If there exists a uniformly positive definite metric

$$
\mathbf{M}(\mathbf{x}, t)=\mathbf{\Theta}(\mathbf{x}, t)^{T} \mathbf{\Theta}(\mathbf{x}, t)
$$

such that the generalized Jacobian

$$
\mathbf{F}=(\dot{\boldsymbol{\Theta}}+\boldsymbol{\Theta} \mathbf{J}) \boldsymbol{\Theta}^{-1}
$$

is uniformly negative definite, then all system trajectories converge exponentially to a single trajectory with convergence rate $\left|\lambda_{\max }\right|$, where $\lambda_{\max }$ is the largest eigenvalue of the symmetric part of $\mathbf{F}$.

Recall that a matrix $\mathbf{A}(\mathbf{x}, t)$ is uniformly positive definite if there exists $\beta>0$ such that

$$
\forall \mathbf{x}, t \quad \lambda_{\min }(\mathbf{A}(t)) \geq \beta
$$




\subsection{Neural networks and locally projected dynamical systems}

Networks of leaky integrators are widely used to model the behavior of neuronal assemblies (Dayan and Abbott, 2001). A leaky integrator network is usually described by the following set of equations

$$
\tau_{i} \dot{x}_{i}=-x_{i}(t)+\sum_{j \neq i} K_{j i} x_{j}(t)+I(t)
$$

where $x(t)$ is the synaptic current of a neuron, $\tau_{i}$ its time constant, $K_{j i}$ the synaptic projection weight from neuron $j$ to neuron $i$ and $I(t)$ the input coming from an external source. Next, $x(t)$ is converted into a non-negative firing rate $y(t)$ using a transfer function, for instance

$$
y(t)=\max (x(t), 0)=[x(t)]_{+}
$$

Another way to enforce nonnegativity of the firing rate can be achieved through locally projected dynamical systems (IPDS in short). These systems were introduced in (Dupuis and Nagurney, 1993) and further analyzed in (Zhang and Nagurney, 1995). Related ideas can be found in the standard parameter projection method in adaptive control (Slotine and Coetsee, 1986; Ioannou and Sun, 1996). A IPDS is given by

$$
\dot{\mathbf{x}}=\Pi_{\Omega}(\mathbf{x}, \mathbf{f}(\mathbf{x}, t))
$$

where $\Omega$ is a convex subset of the state space and $\Pi_{\Omega}$ is the vector-projection operator on $\Omega$ given by

$$
\Pi_{\Omega}(\mathbf{x}, \mathbf{v})=\lim _{h \rightarrow 0^{+}} \frac{\mathbf{P}_{\Omega}(\mathbf{x}+h \mathbf{v})-\mathbf{x}}{h}
$$

In the above equation, $\mathrm{P}_{\Omega}$ denotes the point-projection operator on the convex $\Omega$ 
defined as

$$
\mathbf{P}_{\boldsymbol{\Omega}}(\mathbf{x})=\operatorname{argmin}_{\mathbf{y} \in \mathbf{\Omega}}\|\mathbf{x}-\mathbf{y}\|
$$

Intuitively, if $\mathrm{x}$ is in the interior of $\Omega$ then $\Pi_{\Omega}(\mathrm{x}, \mathrm{v})=\mathrm{v}$. If $\mathrm{x}$ is on the boundary of $\Omega$, then $\Pi_{\Omega}(\mathbf{x}, \mathbf{v})$ is the maximal component of $\mathbf{v}$ that allows the system to remain within $\Omega$. In particular, it is easy to see that any trajectory starting in $\Omega$ remains in $\Omega$.

Note that equation (2) does not define a classical ordinary differential equation since its right-hand side can be discontinuous due to the projection operator. However, under some conditions on $\mathrm{f}$ and $\Omega$ (similar to the Cauchy-Lipschitz conditions for classical ordinary differential equations, see (Dupuis and Nagurney, 1993) and (Filippov, 1963) for more details), existence, uniqueness and some qualitative properties can be established for the solutions of (2). For our purpose, we recall here that any solution $\mathrm{x}$ of (2) is continuous and right-differentiable for all $t$. In the rest of this article, we make the additional assumption that the set of time instants when $\mathbf{x}(t)$ is not differentiable has measure zero.

Within the above framework, the dynamics of a neural network can now be given in matrix form as

$$
\dot{\mathbf{x}}=\Pi_{\mathbb{H}_{n}}(\mathbf{x}, \mathbf{W} \mathbf{x}+\mathbf{I}(t))
$$

where $\mathbf{x}(t)=\left(x_{1}(t), \ldots, x_{n}(t)\right)^{T}$ is the states of the neurons, $\mathbf{W}$ is the $n \times n$ matrix whose diagonal elements represent the leaking rate of the neurons and whose nondiagonal elements represent the synaptic projection weight, $\mathbf{I}(t)$ is the vector of external inputs. Finally, $\mathbb{H}_{n}$ is a regular $n$-cube defined as follows

Definition $1 A$ regular $n$-cube $\mathbb{H}_{n}$ is a subset of $\mathbb{R}^{n}$ defined by

$$
\mathbb{H}_{n}=\left\{\left(x_{1}, \ldots, x_{n}\right) \in \mathbb{R}^{n}: \forall i, m_{i} \leq x_{i} \leq M_{i}\right\}
$$


where $m_{1}, \ldots, m_{n}, M_{1}, \ldots, M_{n} \in \mathbb{R}$.

Intuitively, a regular $n$-cube is a $n$-cube whose edges are parallel to the axes.

In practice, networks of leaky integrators described by IPDS as above and their classical counterparts with transfer functions show very similar behavior. However, the stability properties of IPDS networks can be rigorously established through contraction theory (see next section), which makes them interesting from a theoretical viewpoint.

\subsection{Contraction analysis of locally projected dynamical system on regular n-} cubes

Contraction analysis for systems subject to convex constraints has already been discussed in Lohmiller and Slotine (2000). However, in that work, the projection applied to constrain the system in the convex region depends on the metric which makes the original system contracting. Thus, we cannot expect to use this result here as our projection operator must not depend on the neural network

Since the contraction condition is local, a IPDS can only be contracting if the original, un-projected, system is contracting within $\Omega$. The converse implication is not true in general, because the projection operator can deeply modify the system's behavior along the boundary of $\Omega$. We now introduce a few definitions in order to be able to state this converse implication in some particular cases.

Definition 2 Let $\mathrm{x} \in \delta \boldsymbol{\Omega}$ where $\delta \boldsymbol{\Omega}$ denotes the boundary of $\boldsymbol{\Omega}$. The set of inward normals to $\Omega$ at $\mathrm{x}$ is defined as

$$
N_{\boldsymbol{\Omega}}(\mathbf{x})=\left\{\mathbf{n} \mid \mathbf{n}^{T}(\mathbf{x}-\mathbf{y}) \leq 0, \forall \mathbf{y} \in \boldsymbol{\Omega}\right\}
$$


If $\mathbf{x} \in \Omega-\delta \Omega$ then we set $N_{\Omega}(\mathbf{x})=\{0\}$.

Definition 3 A metric $\mathbf{M}$ is said to be compatible with a convex set $\Omega$ if there exists a coordinate transform $\Theta$ such that $\Theta^{T} \boldsymbol{\Theta}=\mathbf{M}$ and

$$
\forall \mathbf{x} \in \delta \Omega, \forall \mathbf{n} \in N_{\boldsymbol{\Omega}}(\mathbf{x}) \quad \Theta \mathbf{n} \in N_{\Theta \Omega}(\Theta \mathbf{x})
$$

In this case, we say that $\Theta$ is a square-root of $\mathrm{M}$ which is compatible with $\Omega$.

We can give a simple sufficient condition for a metric to be compatible with a regular $n$-cube.

Proposition 1 Any diagonal positive definite metric $\mathbf{M}$ is compatible with any regular n-cube $\mathbb{H}_{n}$.

Proof Let $\mathbf{x}=\left(x_{1}, \ldots, x_{n}\right)^{T} \in \delta \mathbb{H}_{n}$. An inward normal $\mathbf{n}=\left(n_{1}, \ldots, n_{n}\right)^{T}$ to $\mathbb{H}_{n}$ at $\mathrm{x}$ is characterized by

$$
\begin{cases}n_{i} \geq 0 & \text { if } x_{i}=m_{i} \\ n_{i} \leq 0 & \text { if } x_{i}=M_{i} \\ n_{i}=0 & \text { if } m_{i}<x_{i}<M_{i}\end{cases}
$$

Since $\mathbf{M}$ is diagonal and positive definite, one has $\mathbf{M}=\operatorname{diag}\left(d_{1}^{2}, \ldots, d_{n}^{2}\right)$ with $d_{i}>0$. Consider the coordinate transform $\boldsymbol{\Theta}=\operatorname{diag}\left(d_{1}, \ldots, d_{n}\right)$. Clearly, $\boldsymbol{\Theta}^{T} \boldsymbol{\Theta}=$ $\mathbf{M}$ and $\Theta \mathbb{H}_{n}$ is a regular $n$-cube with minimal values $d_{1} m_{1}, \ldots, d_{n} m_{n}$ and maximal values $d_{1} M_{1}, \ldots, d_{n} M_{n}$. It follows from the characterization above that $\boldsymbol{\Theta n}=$ $\left(\mathrm{d}_{1} n_{1}, \ldots, d_{n} n_{n}\right)^{T} \in N_{\boldsymbol{\Theta} \mathbb{H}_{n}}(\boldsymbol{\Theta} \mathbf{x})$

We also need another elementary result. 
Lemma 1 Let $\mathbf{x} \in \Omega$ and $\mathbf{v} \in \mathbb{R}^{n}$. There exists $\mathbf{n}(\mathbf{x}, \mathbf{v}) \in N_{\boldsymbol{\Omega}}(\mathbf{x})$ such that

$$
\Pi_{\Omega}(\mathbf{x}, \mathbf{v})=\mathbf{v}+\mathbf{n}(\mathbf{x}, \mathbf{v})
$$

Proof Let $\mathbf{y} \in \boldsymbol{\Omega}$. We need to show that $A_{\mathbf{y}}=\left(\boldsymbol{\Pi}_{\boldsymbol{\Omega}}(\mathbf{x}, \mathbf{v})-\mathbf{v}\right)^{T}(\mathbf{x}-\mathbf{y}) \leq 0$. By definition of $\Pi_{\Omega}$, one has

$$
A_{\mathbf{y}}=\lim _{h \rightarrow 0^{+}} \frac{1}{h}\left(\mathbf{P}_{\boldsymbol{\Omega}}(\mathbf{x}+h \mathbf{v})-(\mathbf{x}+h \mathbf{v})\right)^{T}(\mathbf{x}-\mathbf{y})
$$

Next, introduce the terms $\mathbf{P}_{\boldsymbol{\Omega}}(\mathbf{x}+h \mathbf{v})$ and $h \mathbf{v}$ into $(\mathbf{x}-\mathbf{y})$

$$
\begin{aligned}
A_{\mathbf{y}}=\lim _{h \rightarrow 0^{+}} \frac{1}{h}[ & \left(\mathbf{P}_{\boldsymbol{\Omega}}(\mathbf{x}+h \mathbf{v})-(\mathbf{x}+h \mathbf{v})\right)^{T}\left(\mathbf{P}_{\boldsymbol{\Omega}}(\mathbf{x}+h \mathbf{v})-\mathbf{y}\right)+ \\
& \left(\mathbf{P}_{\boldsymbol{\Omega}}(\mathbf{x}+h \mathbf{v})-(\mathbf{x}+h \mathbf{v})\right)^{T}\left(\mathbf{x}+h \mathbf{v}-\mathbf{P}_{\boldsymbol{\Omega}}(\mathbf{x}+h \mathbf{v})\right)+ \\
& \left.\left(\mathbf{P}_{\boldsymbol{\Omega}}(\mathbf{x}+h \mathbf{v})-(\mathbf{x}+h \mathbf{v})\right)^{T}(-h \mathbf{v})\right]
\end{aligned}
$$

The first term in the above equation is non-positive by property of the point-projection operator. The second term is the negative of a distance and thus is also non-positive. As for the third term, observe that

$$
\lim _{h \rightarrow 0^{+}}\left(\mathbf{P}_{\boldsymbol{\Omega}}(\mathbf{x}+h \mathbf{v})-(\mathbf{x}+h \mathbf{v})\right)^{T} \mathbf{v}=\left(\mathbf{P}_{\boldsymbol{\Omega}}(\mathbf{x})-\mathbf{x}\right)^{T} \mathbf{v}=0
$$

since $\mathrm{x} \in \Omega$.

We can now state the following theorem

Theorem 2 Let $\dot{\mathbf{x}}=\mathbf{f}(\mathbf{x}, t)$ be a dynamical system which is contracting in a constant metric $\mathbf{M}$ compatible with a convex set $\Omega$. Then the lPDS $\dot{\mathbf{x}}=\Pi_{\Omega}(\mathbf{x}, \mathbf{f}(\mathbf{x}, t))$ is also contracting in the same metric and with the same contraction rate.

Proof Let $\Theta$ be a square-root of $M$ compatible with $\Omega$. Consider $\mathbf{z}=\Theta \mathbf{x}$. By 
lemma 1 , the system $\mathbf{z}$ is described by

$$
\dot{\mathbf{z}}=\Theta \Pi_{\Omega}(\mathbf{x}, \mathbf{f}(\mathbf{x}))=\mathbf{F}(\mathbf{z})+\Theta \mathbf{n}(\mathbf{x}, \mathbf{f}(\mathbf{x}))
$$

where $\mathbf{F}(\mathbf{z})=\boldsymbol{\Theta} \mathbf{f}\left(\Theta^{-1} \mathbf{z}\right)$.

Consider two particular trajectories of (4) $\mathbf{z}_{1}$ and $\mathbf{z}_{2}$. Denote by $\Delta$ the squared distance between $\mathbf{z}_{1}$ and $\mathbf{z}_{2}$

$$
\Delta(t)=\left\|\mathbf{z}_{1}(t)-\mathbf{z}_{2}(t)\right\|^{2}=\left(\mathbf{z}_{1}(t)-\mathbf{z}_{2}(t)\right)^{T}\left(\mathbf{z}_{1}(t)-\mathbf{z}_{2}(t)\right)
$$

When $\Delta$ is differentiable, we have

$$
\begin{aligned}
\frac{d}{d t} \Delta & =2\left(\mathbf{z}_{1}-\mathbf{z}_{2}\right)^{T}\left(\dot{\mathbf{z}}_{1}-\dot{\mathbf{z}}_{2}\right) \\
& =2\left(\mathbf{z}_{1}-\mathbf{z}_{2}\right)^{T}\left(\mathbf{F}\left(\mathbf{z}_{1}\right)+\boldsymbol{\Theta} \mathbf{n}\left(\mathbf{x}_{1}, \mathbf{f}\left(\mathbf{x}_{1}\right)\right)-\left(\mathbf{F}\left(\mathbf{z}_{2}\right)+\boldsymbol{\Theta n}\left(\mathbf{x}_{2}, \mathbf{f}\left(\mathbf{x}_{2}\right)\right)\right)\right)
\end{aligned}
$$

Since the metric is compatible with $\boldsymbol{\Omega}, \boldsymbol{\Theta} \mathbf{n}\left(\mathbf{x}_{i}, \mathbf{f}\left(\mathbf{x}_{i}\right)\right) \in N_{\Theta \Omega}\left(\mathbf{z}_{i}\right)$ for $i=1,2$. Next, by definition of inward normals, we have $\left(\mathbf{z}_{1}-\mathbf{z}_{2}\right)^{T} \boldsymbol{\Theta n}\left(\mathbf{x}_{1}, \mathbf{f}\left(\mathbf{x}_{1}\right)\right) \leq 0$ and $-\left(\mathbf{z}_{1}-\mathbf{z}_{2}\right)^{T} \boldsymbol{\Theta n}\left(\mathbf{x}_{2}, \mathbf{f}\left(\mathbf{x}_{2}\right)\right) \leq 0$, from which we deduce

$$
\begin{aligned}
\frac{d}{d t} \Delta & \left.\leq 2\left(\mathbf{z}_{1}-\mathbf{z}_{2}\right)^{T}\left(\mathbf{F}\left(\mathbf{z}_{1}\right)-\mathbf{F}\left(\mathbf{z}_{2}\right)\right)\right) \\
& \leq-2 \lambda \Delta(t)
\end{aligned}
$$

where $\lambda>0$ is the contraction rate of $\mathbf{f}$ in the metric $\mathbf{M}$.

Since the set of time instants when $\Delta(t)$ is not differentiable has measure zero (see section 2.2), one has

$$
\forall t \geq 0, \Delta(t)=\int_{0}^{t}\left(\frac{d}{d t} \Delta\right) d t \leq-2 \lambda \int_{0}^{t} \Delta(s) d s
$$

which yields by Grönwall's lemma

$$
\forall t \geq 0, \Delta(t) \leq \Delta(0) e^{-2 \lambda t}
$$


i.e.

$$
\forall t \geq 0,\left\|\mathbf{z}_{1}(t)-\mathbf{z}_{2}(t)\right\| \leq\left\|\mathbf{z}_{1}(0)-\mathbf{z}_{2}(0)\right\| e^{-\lambda t}
$$

\subsection{Combination of contracting systems}

One of our motivations for using contraction theory is that contraction properties are preserved under suitable combinations (Lohmiller and Slotine, 1998). This allows both stable aggregation of contracting systems, and variation or optimization of individual subsystems while preserving overall functionality (Slotine and Lohmiller, 2001). We present here three standard combinations of contracting systems which preserve both contraction of the system and diagonality of the metric. Then, constructing our neural network as a IPDS using only those three combinations will give rise to a contracting system in a diagonal metric.

\subsubsection{Negative feedback combination}

Consider two coupled systems

$$
\begin{aligned}
& \dot{\mathbf{x}}_{1}=\mathbf{f}_{1}\left(\mathbf{x}_{1}, \mathbf{x}_{2}, t\right) \\
& \dot{\mathbf{x}}_{2}=\mathbf{f}_{2}\left(\mathbf{x}_{1}, \mathbf{x}_{2}, t\right)
\end{aligned}
$$

Assume that system $i(i=1,2)$ is contracting with respect to $\mathbf{M}_{i}=\boldsymbol{\Theta}_{i}^{T} \mathbf{\Theta}_{i}$, with rate $\lambda_{i}$. Assume furthermore that the two systems are connected by negative feedback (Tabareau and Slotine, 2006). More precisely, the Jacobian matrices of the couplings verify

$$
\Theta_{1} \mathbf{J}_{12} \Theta_{2}^{-1}=-k \Theta_{2} \mathbf{J}_{21}^{\top} \Theta_{1}^{-1}
$$


with $k$ a positive constant. Hence, the Jacobian matrix of the unperturbed global system is given by

$$
\mathbf{J}=\left(\begin{array}{cc}
\mathbf{J}_{1}-k \Theta_{1}^{-1} \Theta_{2} \mathbf{J}_{21}^{\top} \Theta_{1}^{-1} \Theta_{2} \\
\mathbf{J}_{21} & \mathbf{J}_{2}
\end{array}\right)
$$

Consider the coordinate transform

$$
\Theta=\left(\begin{array}{cc}
\Theta_{1} & 0 \\
0 & \sqrt{k} \Theta_{2}
\end{array}\right)
$$

associated to the metric $\mathbf{M}=\boldsymbol{\Theta}^{T} \boldsymbol{\Theta}>\mathbf{0}$. After some calculations, one has

$$
\begin{aligned}
\left(\mathbf{\Theta J} \Theta^{-1}\right)_{s} & =\left(\begin{array}{cc}
\left(\boldsymbol{\Theta}_{1} \mathbf{J}_{1} \boldsymbol{\Theta}_{1}^{-1}\right)_{s} & 0 \\
0 & \left(\boldsymbol{\Theta}_{2} \mathbf{J}_{2} \Theta_{2}^{-1}\right)_{s}
\end{array}\right) \\
& \leq \max \left(-\lambda_{1},-\lambda_{2}\right) \mathbf{I}
\end{aligned}
$$

The augmented system is thus contracting with respect to the metric $\mathbf{M}$, with rate $\min \left(\lambda_{1}, \lambda_{2}\right)$.

\subsubsection{Hierarchical combination}

We first recall a standard result in matrix analysis (Horn and Johnson, 1985). Let A be symmetric matrix in the form

$$
\mathbf{A}=\left(\begin{array}{ll}
\mathbf{A}_{1} & \mathbf{A}_{21}^{T} \\
\mathbf{A}_{21} & \mathbf{A}_{2}
\end{array}\right)
$$


Assume that $\mathbf{A}_{1}$ and $\mathbf{A}_{2}$ are definite positive. Then $\mathbf{A}$ is definite positive if

$$
\sigma^{2}\left(\mathbf{A}_{21}\right)<\lambda_{\min }\left(\mathbf{A}_{1}\right) \lambda_{\min }\left(\mathbf{A}_{2}\right)
$$

where $\sigma\left(\mathbf{A}_{21}\right)$ denotes the largest singular value of $\mathbf{A}_{21}$. In this case, the smallest eigenvalue of A satisfies

$$
\lambda_{\min }(\mathbf{A}) \geq \frac{\lambda_{\min }\left(\mathbf{A}_{1}\right)+\lambda_{\min }\left(\mathbf{A}_{2}\right)}{2}-\sqrt{\left(\frac{\lambda_{\min }\left(\mathbf{A}_{1}\right)-\lambda_{\min }\left(\mathbf{A}_{2}\right)}{2}\right)^{2}+\sigma^{2}\left(\mathbf{A}_{21}\right)}
$$

Consider now the same set-up as in section 2.4.1, except that the connection is now hierarchical and upper-bounded. More precisely, the Jacobians of the couplings verify

$$
\mathbf{J}_{12}=\mathbf{0}, \quad \sigma^{2}\left(\Theta_{2} \mathbf{J}_{21} \Theta_{1}^{-1}\right) \leq K
$$

Hence, the Jacobian matrix of the augmented system is given by

$$
\mathbf{J}=\left(\begin{array}{ll}
\mathbf{J}_{1} & \mathbf{0} \\
\mathbf{J}_{21} & \mathbf{J}_{2}
\end{array}\right)
$$

Consider the coordinate transform

$$
\boldsymbol{\Theta}_{\epsilon}=\left(\begin{array}{cc}
\boldsymbol{\Theta}_{1} & 0 \\
0 & \epsilon \\
\Theta_{2}
\end{array}\right)
$$

associated to the metric $\mathbf{M}_{\epsilon}=\boldsymbol{\Theta}_{\epsilon}^{T} \boldsymbol{\Theta}_{\epsilon}>\mathbf{0}$. After some calculations, one has

$$
\left(\boldsymbol{\Theta} \mathbf{J} \Theta^{-1}\right)_{s}=\left(\begin{array}{cc}
\left(\boldsymbol{\Theta}_{1} \mathbf{J}_{1} \boldsymbol{\Theta}_{1}^{-1}\right)_{s} & \frac{1}{2} \epsilon\left(\boldsymbol{\Theta}_{2} \mathbf{J}_{21} \boldsymbol{\Theta}_{1}^{-1}\right)^{T} \\
\frac{1}{2} \epsilon \boldsymbol{\Theta}_{2} \mathbf{J}_{21} \boldsymbol{\Theta}_{1}^{-1} & \left(\boldsymbol{\Theta}_{2} \mathbf{J}_{2} \boldsymbol{\Theta}_{2}^{-1}\right)_{s}
\end{array}\right)
$$


Set now $\epsilon=\sqrt{\frac{2 \lambda_{1} \lambda_{2}}{K}}$. The augmented system is then contracting with respect to the metric $\mathbf{M}_{\epsilon}$, with rate $\lambda$ verifying

$$
\left.\lambda \geq \frac{1}{2}\left(\lambda_{1}+\lambda_{2}-\sqrt{\lambda_{1}^{2}+\lambda_{2}^{2}}\right)\right)
$$

\subsubsection{Small gains}

In this section, we require no specific assumption on the form of the couplings

$$
\mathbf{J}=\left(\begin{array}{ll}
\mathbf{J}_{1} & \mathbf{J}_{12} \\
\mathbf{J}_{21} & \mathbf{J}_{2}
\end{array}\right)
$$

As for negative feedback, consider the coordinate transform

$$
\Theta_{k}=\left(\begin{array}{cc}
\Theta_{1} & 0 \\
0 & \sqrt{k} \Theta_{2}
\end{array}\right) \quad k>0
$$

associated to the metric $\mathbf{M}_{k}=\Theta_{k}^{T} \Theta_{k}>0$. After some calculations, one has

$$
\left(\boldsymbol{\Theta}_{k} \mathbf{J} \Theta_{k}^{-1}\right)_{s}=\left(\begin{array}{cc}
\left(\Theta_{1} \mathbf{J}_{1} \Theta_{1}^{-1}\right)_{s} & \mathbf{A}_{k}^{T} \\
\mathbf{A}_{k} & \left(\boldsymbol{\Theta}_{2} \mathbf{J}_{2} \Theta_{2}^{-1}\right)_{s}
\end{array}\right)
$$

where $\mathbf{A}_{k}=\frac{1}{2}\left(\sqrt{k} \Theta_{2} \mathbf{J}_{21} \Theta_{1}^{-1}+\frac{1}{\sqrt{k}}\left(\Theta_{1} \mathbf{J}_{12} \Theta_{2}^{-1}\right)^{T}\right)$. Following the result stated at the beginning of section 2.4.2, if

$$
\min _{k} \sigma^{2}\left(\mathbf{A}_{k}\right)<\lambda_{1} \lambda_{2}
$$


then the augmented system is contracting with respect to the metric $\mathbf{M}_{k}$ for some $k$, with rate $\lambda$ verifying

$$
\lambda \geq \frac{\lambda_{1}+\lambda_{2}}{2}-\sqrt{\left(\frac{\lambda_{1}-\lambda_{2}}{2}\right)^{2}+\min _{k} \sigma^{2}\left(\mathbf{A}_{k}\right)}
$$

\section{Model description}

Rather than using standard leaky-integrator rate-coding neurons, we use the very similar local projected dynamical system model defined by equation 3 , where each component of the state vector $\mathbf{x}$ is an artificial rate-coding neuron representing the discharge rate of populations of real neurons. Each competing BG channel in each nucleus is represented by one such neuron, and the corresponding thalamic nucleus and cortical areas are also subdivided in identical channels (Fig. B.2). The convergence of cortical sensory inputs on the striatum channels is encoded, for simplicity, by a vector of saliences (one salience per channel). Each salience represents the propensity of its corresponding channel to be selected. Each behavior in competition is associated to a specific channel and can be executed if and only if its level of inhibition decreases below a the inhibition level at rest $y_{\text {Rest }}^{G P i}$ (ie. the $\mathrm{SNr} / \mathrm{GPi}$ output when the salience vector is null).

[Fig. 2 about here.]

The main difference of our architecture with the recent GPR proposal (Gurney et al., 2001a) is the nuclei targeted by the external part of the globus pallidus (GPe) and the nature of these projections. In our model, the GPe projects to the subthalamic nucleus (STN), the internal part of the globus pallidus (GPi) and the substantia nigra pars reticulata $(\mathrm{SNr})$, as well as to the striatum, as documented in (Staines et al., 1981; Bevan et al., 1998; Kita et al., 1999). Moreover, the striatal terminals 
target the dendritic trees, while pallidal, nigral and subthalamic terminals form perineuronal nets around the soma of the targeted neurons (Sato et al., 2000). This specific organization allows GPe neurons to influence large sets of neurons in GPi, SNr and STN (Parent et al., 2000), thus the sum of the activity of all GPe channels influences the activity of STN and GPi/SNr neurons (equation 9 and 11), while there is a simple channel-to-channel projection to the striatum (equation 6 and 7).

The striatum is one of the two input nuclei of the BG. It is mainly composed of GABAergic (inhibitory) medium spiny neurons (MSN). As in the GPR model, we distinguish among them, those with D1 and D2 dopamine receptors and modulate the input generated in the dendritic tree by the dopamine level $\gamma$, which here encompasses salience, frontal cortex feedback and GPe projections.

Using the formulation of equation 3 , the $i$ th neuron $(i \in[1, N]$, with $N$ the number of channels) of the D1 and D2 sub parts of the striatum are defined as follows

$$
\begin{aligned}
(\mathbf{W} \mathbf{x}+\mathbf{I}(t))_{D 1_{i}}= & \frac{1}{\tau}\left((1+\gamma)\left(w_{F C}^{D 1} x_{i}^{F C}-w_{G P e}^{D 1} x_{i}^{G P e}+w_{S}^{D 1} S_{i}(t)\right)\right. \\
& \left.-w_{F S}^{D 1} x^{F S}+I_{D 1}\right) \\
(\mathbf{W} \mathbf{x}+\mathbf{I}(t))_{D 2_{i}}= & \frac{1}{\tau}\left((1-\gamma)\left(w_{F C}^{D 2} x_{i}^{F C}-w_{G P e}^{D 2} x_{i}^{G P e}+w_{S}^{D 2} S_{i}(t)\right)\right. \\
& \left.-w_{F S}^{D 2} x^{F S}+I_{D 2}\right)
\end{aligned}
$$

where $S(t)$ is the salience input vector, and where the negative constant input $I_{D 1}$ and $I_{D 2}$, which keep the neurons silent when the inputs are not strong enough, model the up-state/down-state property of the MSNs.

The striatum also contains a small proportion of phenotypically diverse interneurons (Tepper and Bolam, 2004). We include here the fast spiking GABAergic interneurons (FS), that we model roughly as single population exerting feedforward 
inhibition on the MSN (Tepper et al., 2004), and modulated by GPe feedback (Bevan et al., 1998)

$$
(\mathbf{W} \mathbf{x}+\mathbf{I}(t))_{F S}=\frac{1}{\tau_{F S}} \sum_{j=1}^{N}\left(w_{F C}^{F S} x_{j}^{F C}-w_{G P e}^{F S} x_{j}^{G P e}+w_{S}^{F S} S_{j}(t)\right)
$$

The sub-thalamic nucleus (STN) is the second input of the basal ganglia and also receives diffuse projections from the GPe, as explained above. Its glutamatergic neurons have an excitatory effect and project to the GPe and GPi. The resulting input of the STN neuron is given by

$$
(\mathbf{W} \mathbf{x}+\mathbf{I}(t))_{S T N_{i}}=\frac{1}{\tau_{S T N}}\left(w_{F C}^{S T N} x_{i}^{F C}-w_{G P e}^{S T N} \sum_{j=1}^{N} x_{j}^{G P e}+I_{S T N}\right)
$$

where the constant positive input $I_{S T N}$ models the tonic activity of the STN.

The GPe is an inhibitory nucleus, it receives channel-to-channel afferents from the whole striatum (Wu et al., 2000), and a diffuse excitation from the STN

$$
(\mathbf{W} \mathbf{x}+\mathbf{I}(t))_{G P e_{i}}=\frac{1}{\tau}\left(-w_{D 1}^{G P e} x_{i}^{D 1}-w_{D 2}^{G P e} x_{i}^{D 2}+w_{S T N}^{G P e} \sum_{j=1}^{N} x_{j}^{S T N}+I_{G P e}\right)
$$

where the constant positive input $I_{G P e}$ models the tonic activity of the GPe.

The GPi and SNr are the inhibitory output nuclei of the BG, which keep their targets under inhibition unless a channel is selected. They receive channel-to-channel projections from the D1 striatum and diffuse projections from the STN and the GPe

$$
\begin{aligned}
(\mathbf{W} \mathbf{x}+\mathbf{I}(t))_{G P i_{i}}= & \frac{1}{\tau}\left(-w_{D 1}^{G P i} x_{i}^{D 1}+w_{S T N}^{G P i} \sum_{j=1}^{N} x_{i}^{S T N}\right. \\
& \left.-w_{G P e}^{G P i} \sum_{j=1}^{N} x_{j}^{G P e}+I_{G P i}\right)
\end{aligned}
$$

where the constant positive input $I_{G P i}$ models the tonic activity of the GPi/SNr.

Finally, the thalamus (TH) forms an excitatory loop with the frontal cortex (FC), 
these two modules representing different thalamus nuclei and cortical areas, depending on the cortico-baso-thalamo-cortical loop considered. The thalamus is moreover under a global regulatory inhibition of the thalamic reticular nucleus (TRN, represented by a single population of neurons) and a channel-specific selective inhibition from the basal ganglia

$$
\begin{gathered}
(\mathbf{W} \mathbf{x}+\mathbf{I}(t))_{T H_{i}}=\frac{1}{\tau_{T H}}\left(w_{F C}^{T H} x_{i}^{F C}-w_{T R N}^{T H} x^{T R N}-w_{G P i}^{T H} x^{G P i}\right) \\
(\mathbf{W} \mathbf{x}+\mathbf{I}(t))_{F C_{i}}=\frac{1}{\tau_{F C}}\left(w_{S}^{F C} S_{i}+w_{T H}^{F C} x_{i}^{T H}\right) \\
(\mathbf{W} \mathbf{x}+\mathbf{I}(t))_{T R N}=\frac{1}{\tau_{T R N}}\left(\sum_{i} w_{F C}^{T R N} x_{i}^{F C}+w_{T H}^{T R N} x_{i}^{T H}\right)
\end{gathered}
$$

This model keeps the basic off-center on-surround selecting structure, duplicated in the D1-STN-GPi/SNr and D2-STN-GPe sub-circuits, of the GPR. However, the channel specific feedback from the GPe to the Striatum helps sharpening the selection by favoring the channel with the highest salience in D1 and D2. Moreover, the global GPe inhibition on the GPi/SNr synergetically interacts with the STN excitation in order to limit the amplitude of variation of the inhibition of the unselected channels. The inhibitory projections of the BG onto the thalamo-cortical excitatory loop limits the amplification of the unselected channels and thus favors a selective amplification of the winning channels. In such an architecture, the frontal cortex preserves the information from all channels but amplifies selectively the winning channel, in sort of attention "spotlight" process, while the subcortical target circuits of the BG are under very selective inhibition, ensuring that motor commands do not interfere. 


\section{Disembodied model results}

We first analyze the contraction of contracting basal ganglia model (CBG) and its selection properties in simple disembodied tests before evaluating it as an ASM in a simulated robot.

[Table 1 about here.]

Similarly to the simulations made by Gurney et al. (2001b), we used a 6-channel model. The parameters of the model were hand-tuned in order to obtain a selective system and respecting the local contraction constraints defined below, their values are summarized in table B.1. The simulation was programmed in $\mathrm{C}++$, using the simple Euler approximation for integration, with a time step of $1 \mathrm{~ms}$.

\subsection{Contraction analysis of the model}

According to the theory developed in section 2.3, our model is contracting if the non projected dynamics (which is linear) is contracting in a diagonal metric. To find this metric, we will use the three combinations presented in section 2.4 that preserve diagonality.

Remark that each separated nucleus is trivially contracting in the identity metric because there is no lateral connection. The contracting rate of each nucleus is $\frac{1}{\tau}$, where $\tau$ is the common time constant of the $N$ neurons of the nucleus. Thus, the metric $\mathbf{M}_{B G}$ of the basal ganglia is constituted of the blocks $\kappa_{G P e} \mathbf{I}, \kappa_{S T N} \mathbf{I}, \kappa_{D 1} \mathbf{I}$, $\kappa_{D 2} \mathbf{I}, \kappa_{F S} 1$ and $\kappa_{G P i} \mathbf{I}$. Similarly, the thalamic metric $\mathbf{M}_{T H}$ is constituted of the blocks $\kappa_{F C} \mathbf{I}, \kappa_{T H} 1$ and $\kappa_{T R N} \mathbf{I}$. The resulting metric for the whole system $\mathbf{M}_{C B G}$ 
combines $\mathbf{M}_{B G}$ and $\mathbf{M}_{T H}$ in the following way

$$
\mathbf{M}_{C B G}=\left(\begin{array}{cc}
\mathbf{M}_{B G} & 0 \\
0 & \alpha \mathbf{M}_{T H}
\end{array}\right)
$$

\section{Analysis of the basal ganglia.}

- $\kappa_{G P e}=1$

We can set $\kappa_{G P e}$ to any value as there is no combination at this stage. The current contracting rate is $\frac{1}{\tau}$.

- $\kappa_{S T N}=w_{S T N}^{G P e} / w_{G P e}^{S T N}$

We use negative feedback. The contracting rate remains unchanged

- $\left\{\begin{array}{l}\kappa_{D 1}=w_{D 1}^{G P e} /\left((1+\gamma) w_{G P e}^{D 1}\right) \\ \kappa_{D 2}=w_{D 2}^{G P e} /\left((1-\gamma) w_{G P e}^{D 2}\right)\end{array}\right.$

We use small gains to show that the system constituted by the STN, GPe, striatum D1 and D2 is contracting when

$$
\left((1+\gamma) w_{D 1}^{G P e} w_{G P e}^{D 1}\right)^{2}+\left((1-\gamma) w_{D 2}^{G P e} w_{G P e}^{D 2}\right)^{2}<1
$$

with a contracting rate $\frac{1}{\tau}\left(1-\sqrt{\left((1+\gamma) w_{D 1}^{G P e} w_{G P e}^{D 1}\right)^{2}+\left((1-\gamma) w_{D 2}^{G P e} w_{G P e}^{D 2}\right)^{2}}\right)$

- $\kappa_{F S}=w_{F S}^{D 1} / w_{G P e}^{F S}$

Again by use of small gains.

- $\kappa_{G P i}=1 /(\tau \sigma(\mathbf{G}))^{2}$

where $\sigma(\mathbf{G})$ is the largest singular value of the matrix of projections on GPi and $\tau$ is the slowest time constant of neurons in the basal ganglia. This constant is set by using hierarchical combination.

Thus we can guarantee the contraction of the basal ganglia as soon as condition 
(15) is satisfied.

\section{Analysis of the thalamus.}

- $\kappa_{T H}=1$

We can set $\kappa_{T H}$ to any value as there is no combination at this stage. The current contracting rate is $\frac{1}{\tau_{T H}}$.

- $\kappa_{G P e}=w_{T R N}^{T H} / w_{T H}^{T R N}$

We use negative feedback. The contracting rate remains unchanged

- $\kappa_{F C}=\sqrt{w_{F C}^{T H} 2+N w_{F C}^{T R N^{2}}} / w_{T H}^{F C}$

We use small gains to show that the thalamo-cortical module is contracting when

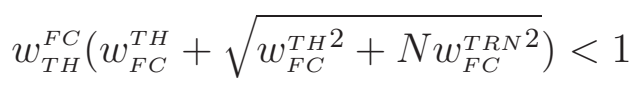

Remark that this condition depends on $N$. This would not have been the case if we have modelled the $T R N$ by $N$ channels instead of 1 .

Thus we can guarantee the contraction of the thalamus as soon as condition (16) is satisfied.

It remains to examine the large loop between the thalamus and the basal ganglia involving projections of the GPi and the FC. Again, we use small gains to set $\alpha$.

$$
\alpha=\sqrt{\frac{\tau_{F C t x} \kappa_{G P i}\left(w_{F C}^{S T N 2}+w_{F C}^{D 1} 2+w_{F C}^{D 2} 2+n w_{F C}^{F S}\right)}{\tau_{T H} \kappa_{F C} w_{G P i}^{T H}{ }^{2}}}
$$

Proposition 3 Let $\mathbf{M}_{C B G}=\Theta_{C B G}^{T} \Theta_{C B G}$ be the diagonal metric defined above. By theorem 2, if the generalised Jacobian $\Theta_{C B G} \mathbf{W} \Theta_{C B G}^{-1}$ is negative definite, the dynamical system $\dot{\mathbf{x}}=\Pi_{\mathbb{H}_{n}}(\mathbf{x}, \mathbf{W} \mathbf{x}+\mathbf{I}(t))$ describing the cortico-baso-thalamocortical loop model is contracting with a rate $\left|\lambda_{\max }\right|$, where $\lambda_{\max }$ is the largest eigenvalue of $\Theta_{C B G} \mathbf{W} \Theta_{C B G}^{-1}$. 
At this stage, we have provided an algebraic definition of the metric $\mathbf{M}_{C B G}$. Unfortunately, the complexity of the induced generalized Jacobian prevents us from giving a global algebraic condition on the projection weights for the generalized Jacobian to be negative definite. This is not of major incidence as we can compute numerically, for any instance of the weights, the eigenvalues of the symmetric part of the generalized Jacobian and check that they are all negative.

Table B.2 gives the numerical value of the constants defining the metric $M_{C B G}$ for the set of parameters of our simulation (see table B.1). Using the free software Octave, we compute in that case the eigenvalues of the generalised Jacobian and obtain that our model is contracting with contracting rate of 2.20 .

Notice that computing the maximum real part of the eigenvalue of the non projected dynamics (which is linear) gives an upper bound of the contracting rate. For the set of parameters of our simulation, this upper bound is 2.59. It is remarkable that being forced to use diagonal metrics in our proof (which discards a huge set of metrics) has not much decreased the contracting rate.

[Table 2 about here.]

\subsection{Basic selection test}

We first reproduced the selection test of Gurney et al. (2001b) with our model and with the GPR model version presented in (Prescott et al., 2006). In this test, a specific sequence of five different salience vectors (represented by the dashed lines in fig. B.3) is submitted to a 6-channels version of the BG model, in order to show the basic selection properties of the system. Here, we submitted each vector to the system during $2 s$ before switching to the next one in the sequence. 
[Fig. 3 about here.]

During the CBG simulation (fig. B.3, top row), with the first vector of null saliences, the system stabilizes in a state where all channels are equally inhibited $\left(x_{i}^{G P i}=\right.$ 0.095). Then, the first channel receives a 0.4 input salience which results in a clear disinhibition of this channel $\left(x_{1}^{G P i}=0.014\right)$ and increased inhibition of the others. When the second channel salience is set to 0.6 , it becomes perfectly selected $\left(x_{2}^{G P i}=0\right)$ while the first one is rapidly inhibited to a level identical to the one of the four last channels. During the fourth step, the salience of the first channel is increased to 0.6 , channels 1 and 2 are therefore simultaneously selected $\left(x_{1}^{G P i}=x_{2}^{G P i}=0.03\right)$. Finally, during the last step of the test, channel 1 has its salience reduced to 0.4 , and it is then rapidly inhibited, while the channel 2 returns to perfect selection $\left(x_{2}^{G P i}=0\right)$. The CBG thus passes this test in a satisfactory manner: the channels with the highest saliences are always selected while the others are inhibited.

The GPR simulation (fig. B.3, bottom row) is qualitatively quite similar, excepted during the fourth step of the sequence (emphasized with an asterisk): while the salience of channel 1 increases from 0.4 up to 0.6 (the same salience as channel 2), channel 2 remains selected and channel 1 is fully inhibited (its level of inhibition is higher than the inhibition at rest). The inputs in channels 1 and 2 being exactly the same, this difference in their selection state is clearly caused by the initial conditions of the system (i.e. the fact that channel 2 was selected before). This example of a dependence on the initial conditions clearly shows that the GPR model is not contracting.

Indeed, as we have seen in section 2.3, a rate coding neural network is contracting only if its non projected dynamics is contracting in a diagonal metric. But a linear 
system is stable if and only if all its eigenvalues have a negative real part. Computing the eigenvalues of the linear part of the GPR reveals that $N-1$ of them have a positive real part (namely 10.387). We can thus conclude that the GPR is not contracting.

\subsection{Systematic salience search test}

This first result is however not surprising, as revealed by the systematic salience search experiment performed in (Prescott et al., 2006), and that we also reproduced with both the GPR and the CBG. In this experiment, the first two channels of the ASM are put in competition in the following manner: the first channel salience is increased from 0 to 1 in steps of 0.01 , and for each of these steps, the salience of the second channel is also gradually increased from 0 to 1 in steps of 0.01 . The system is run to convergence between all step increases. The internal state of the model is not reset between each channel 2 salience increase, but only for channel 1 steps. This means that the test evaluates the selection response of the system with one channel salience fixed while the other one gradually increases.

[Fig. 4 about here.]

In order to evaluate the response of the ASM to this experiment, four numerical values are computed. First, the efficiencies of the selection of channel 1 and 2, equivalent to the percentage of disinhibition, is computed as follows:

$$
e_{i}=\left[1-y_{i}^{G P i} / y_{\text {Rest }}^{G P i}\right]_{+}
$$

with $i$ the index of the channel, $y_{i}^{G P i}$ the output of the $i t h$ GPi neuron and $y_{R e s t}^{G P i}$ the output inhibition of all channels when all saliences are null. The absolute efficiency 
of the selection is defined as the efficiency of the winning channel:

$$
e_{w}=\max _{i} e_{i}
$$

Finally, the distortion of the selection, which is null when the winning channel only is disinhibited and increasing with the disinhibition of its competitors, is defined by:

$$
d_{w}=2 \frac{\sum_{i} e_{i}-e_{w}}{\sum_{i} e_{i}}
$$

The results of the experiment are summarized by the $e_{w}$ and $d_{w}$ graphs (fig. B.4), where the value of the each of these variables is represented with regards to the corresponding channel 1 (abscissa) and channel 2 (ordinate) saliences. First observe that the GPR results we obtain with 6 channels are very similar to those presented in (Prescott et al., 2006) for a 5-channel GPR. Concerning $e_{w}$ (top row), whereas, for the $\mathrm{CBG}$, the selection switches from channel 1 to channel 2 as soon as the salience of channel 2 is larger than the salience of channel 1 (when it crosses the diagonal in dashed black), for the GPR, this switch is delayed until much higher values are reached (when is crosses the black line). As previously noted, this hysteresis effect is a direct consequence of the non-contraction of the GPR.

Note that when high saliences are in competition, the GPR tends to partially select both channels ( $e_{w}<1$ and $d_{w}>0$ ), while the CBG fully disinhibits both channels ( $e_{w}=1$ and $d_{w}$ close to 1 ). Which behavior is preferable for an ASM is not decided. Is the GPR strong dependence on initial conditions a good feature for an ASM? Prescott et al. (2006) argue that it allows behavioral persistence, and that in their experiment, the robot takes advantage of it to avoid dithering between actions. We do not claim that there is a definitive answer to the question. Nevertheless, in the next section, we describe the evaluation of the $\mathrm{CBG}$ in a minimal survival task in which the robot also avoids dithering, despite its contracting ASM. This shows that 
this dependence on initial conditions is not necessary from the point of view of dithering avoidance.

\section{Minimal survival task}

\subsection{Material and methods}

The suitability of the model for action selection in an autonomous robot has been tested in simulation with the same minimal survival task previously used to evaluate the GPR model (Girard et al., 2003). In order to emphasize its properties, and in particular those resulting from the selective feedback loop, its performance was compared to a simple if-then-else decision rule (ITE, fully described in appendix A).

In such a task, the robot has to go back and forth between locations containing two different kind of resources, in order to keep its energy level above 0 . The robot has two internal variables, namely Energy and Potential Energy, taking values between 0 and 1, and an artificial metabolism, which couples them as follows:

- The Energy $(E)$ is continuously decreasing, with a constant consumption rate (0.01 Energy unit per second). When it reaches 0 , the robot has run out of energy and the ongoing trial is interrupted. To prevent this, the robot has to regularly acquire Energy by activating the ReloadOnE action on an Energy resource. Note that ReloadOnE only transforms Potential Energy into Energy (0.2 units of $E_{p}$ are transformed in 0.2 units of $E$ each second), thus Potential Energy has to be also reloaded.

- The Potential Energy $\left(E_{p}\right)$ is a sort of Energy storage, it can be acquired by acti- 
vating the ReloadOnEp action on a Potential Energy resource, and is consumed in the transformation process only.

In this version of the task, the experiments are run in simulation using the Player/Stage robot interface and robot simulator (Gerkey et al., 2003). The simulated robot is a $40 \times 50 \mathrm{~cm}$ wheeled robot with differential steering, similar to the Activ-Media Pioneer 2DX (fig. B.5), equipped with a ring of 16 sonars and a camera. The sonar sensors have a maximum range of $5 \mathrm{~m}$ and a view angle of $15^{\circ}$, the camera has a resolution of $200 \times 40$ pixels and a view angle of $60^{\circ}$ and uses a color-blob-finding vision device to track the position of red and blue objects. The experiment takes place in a $10 \times 10 \mathrm{~m}$ arena, containing one Energy and one Potential Energy resource (fig. B.5). These resources are represented by colored $50 \times 50 \mathrm{~cm}$ objects (respectively red and blue), and don't constitute obstacles (as if they were suspended above the arena). They are randomly positioned in the arena for each trial, with the constraint that their center is at least $1 m$ away from the walls.

[Fig. 5 about here.]

The robot has to select among seven possible actions:

- ReloadOnE $(R O E)$ and ReloadOnEp $\left(R O E_{p}\right)$ affect the robot's survival as previously described. These actions are effective if the robot is facing the corresponding resource and is close enough $\left(45^{\circ}\right.$ of the camera field of view is occupied by the resource).

- Wander $(W)$ activates random accelerations, decelerations and turning movements.

- Rest $(R)$ stops the robot, which is a disadvantage as the robot has to continuously explore the arena to find resources, but Rest also halves the rate of Energy consumption (0.005 unit per second), which promotes long survival. Consequently, 
it should be activated when there is no risk (i.e. when both internal variables reach high levels) in order to minimize the Potential Energy extracted from the environment to survive.

- AvoidObstacle $(A O)$ uses data from the 6 front sonars the 2 central rear sonars in order to avoid collisions with walls.

- ApproachE $(A E)$ and ApproachEp $\left(A E_{p}\right)$ use the color-blob-finder in order to orient and displace the robot towards the corresponding resource if it is visible.

The action selection mechanisms base their decisions on the following variables:

- $E, E_{p},(1-E)$ and $\left(1-E_{p}\right)$, which provide the amount (or lack of) Energy and Potential Energy,

- seeEBlob and seeEpBlob, which are set to 1 if a red (resp. blue) object is in the camera input, and to 0 otherwise,

- $o n E B l o b$ and $o n E p B l o b$, which are set to 1 if a red (resp. blue) object is larger than 150 pixels (i.e. close enough to allow the use of the corresponding resource), and to 0 otherwise,

- $S F R$ and $S F L$ are the values of the front-right and front-left sonar sensors, measured in meters, taking values between 0 and 5 .

For the $\mathrm{CBG}$, the detailed salience computation using these variables is given in appendix B.

The action selection mechanisms receive new sensory data every $100 \mathrm{~ms}$, and must then provide an action selection for the next $100 \mathrm{~ms}$. Concerning the ITE, it is simply done by executing the decision rule once with the latest data. Concerning the $\mathrm{CBG}$, the selection is made using the output inhibition resulting from the computation of 100 simulation steps of $1 \mathrm{~ms}$, using the latest sensory data. A given action is then considered selected if the inhibition of the corresponding channel is below 
the inhibition at rest $y_{\text {Rest }}^{G P i}$ (as defined previously). In the case of multiple channel disinhibition, the following action combination rules have been defined:

- Rest is effective if and only if it is the only disinhibited action,

- ReloadOnE and ReloadOnEp are effective if and only if the robot does not move,

- The other movement-generating actions can be co-activated. In that case, the efficiency of selection (as defined by equation 17) is used to weight the contributions of each action to the final motor command.

The comparison between the CBG and the ITE is made according to the following protocol: 20 random resource positions are drawn and, for each model, 20 trials are run using the same set of positions. The robot begins the experiment with a full battery $(E=1)$ and no Potential Energy storage $\left(E_{p}=0\right)$, this allows a maximal survival duration of 1 min40s if no reloading action occurs. Unless the robot runs out of energy $(E=0)$, the trial is stopped after $15 \mathrm{~min}$.

\subsection{Results}

The first result is that the CBG and the ITE algorithm have similar survival performance. They are both able to survive the trial in a majority of cases, but can be subject to premature Energy shortage. This is expected, because their ability to find resources is limited by the camera range and field of view, as well as by the random exploration action. The average survival duration is $687 s(\sigma=244)$ for the CBG and $737 s(\sigma=218)$ for the ITE, and the two-tailed Kolmogorov-Smirnov test confirms that the two sets of survival durations are not drawn from significantly different distributions $\left(D_{K S}=0.2, p=0.771\right)$. From an action selection point of view, the comparison of the two mechanisms is thus fair: despite they were tuned 
independently, they both achieve similar survival performance.

[Fig. 6 about here.]

Nevertheless, a clear behavioral difference between the two mechanisms was observed, which has significant repercussions on their ability to store Potential Energy and on the Potential Energy extracted from the environment. Indeed, while the CBG may use its feedback loops in order to persist in action execution, the ITE was deliberately deprived of any memory. This was done in order to investigate the effects of this persistence property. The ITE exhibits behavioral dithering in a critical and frequent situation: when the robot fully reloads its Energy, it activates the Wander action, but after $100 \mathrm{~ms}$ of Wander execution, some Energy has been consumed and the robot has not moved much. In most cases, it is still on the Energy resource, and if it still has spare $E_{p}$, ReloadOnE is activated again. This repeats until there is no $E_{p}$ left or until, small movements by small movements, the robot has left the resource (see fig. B.6). This dithering generates a strong energy dissipation: $100 \mathrm{~ms}$ of Wander consumes 0.001 units of Energy, and during the following $100 \mathrm{~ms}$, ReloadOnEnergy consumes 0.02 units of $E_{p}$ while $E$, being bounded by 1 , increases of 0.001 only.

On the contrary, in the same situation, the CBG takes advantage of a hysteresis effect caused by the positive feedback from the frontal cortex to the basal ganglia to avoid dithering.

[Fig. 7 about here.]

Indeed, the salience of ROE is defined by: $S_{R O E}=950 \times f\left(4 \times\right.$ onEBlob $\times E_{p} \times$ $(1-E))+0.6 \times x_{R O E}^{F C}$ (where $f$ is a sigmoid transfer function, see appendix B). Consequently, when the robot has a lack of Energy and reaches an Energy resource, 
onE Blob jumps from 0 to 1 and $S_{R O E}$ also jumps from 0 (fig. B.7, point A) to a level depending on the current $E$ and $E_{p}$ internal states (fig. B.7, point B) situated on the raw $S_{R O E}$ curve (fig. B.7, dashed line). In the case depicted in fig. B.7, $S_{R O E}$ is then much higher than $S_{W}$, and ROE is thus selected. As a consequence, the corresponding thalamo-cortical channel is disinhibited, leading to an amplification of the salience, fed back to the basal ganglia thanks to the cortical output $x_{R O E}^{F C}$ (this bonus is represented by the shaded area over the raw $S_{R O E}$ curve on fig. B.7). While the robot reloads, $S_{R O E}$ decreases with $\left(E_{p} \times(1-E)\right)$, but because of the $x_{R O E}^{F C}$ salience bonus, it follows the blue trajectory down to point $\mathrm{C}$, where Wander is selected again. The deselection of ROE shuts off the $x_{R O E}^{F C}$ signal, causing an immediate decrease to point D. As soon as the robot activates Wander, Energy is consumed and $S_{R O E}$ increases again, along the raw $S_{R O E}$ curve. However, at point D, $S_{R O E}<S_{W}$, and as long as the robot manages to leave the resource before $S_{R O E}$ exceeds $S_{W}$ (points E and F, when the $O n E B l o b$ variable jumps from 1 to $0)$, no dithering occurs.

[Fig. 8 about here.]

This observation is not trivial, as it has a direct consequence on the global $E_{p}$ storage of the ITE: both CBG and ITE keep high levels of $E_{p}$ (between 0.9 and 1) more than $50 \%$ of the time (fig. B.8, right), but for the rest of the time, the ITE level is very low $(0-0.1)$ much more often (almost $20 \%$ of the time) than the CBG. Moreover, the $\mathrm{CBG}$ activates the Rest action often enough to extract, on average, less Potential Energy from the environment $\left(0.93 \times 10^{-2}\right.$ Ep.s $\left.^{-1}, \sigma=0.30 \times 10^{-3}\right)$ than the basic rate $\left(1 \times 10^{-2} E p . s^{-1}\right)$. On the contrary, the dissipation of energy caused by the dithering of the ITE generates a much higher Potential Energy extraction rate $\left(1.17 \times 10^{-2}\right.$ Ep.s $\left.s^{-1}, \sigma=1.17 \times 10^{-3}\right)$. The two-tailed Kolmogorov-Smirnov test reveals that the $E_{p}$ consumption rates measured for the CBG and the ITE (fig. B.9) 
are drawn from different distributions $\left(D_{K S}=0.95, p<0.001\right)$. The ITE dithering thus generates so much dissipation that it has to extract extra Potential Energy from the environment, despite its use of the Sleep action to lower its consumption, while the $\mathrm{CBG}$ exploits as much as possible this possibility to limit Potential Energy extraction.

[Fig. 9 about here.]

\section{Discussion}

We proposed a new action selection mechanism for an autonomous robot, using a multidisciplinary approach combining computational neuroscience and dynamic system theory. This study proved fruitful in the three considered domains:

- We proposed an extension of the contraction theory to locally projected dynamical systems, which was necessary to study the stability of rate-coding neural networks.

- As a consequence, we proposed a modified rate-coding artificial neuron model.

- Using these results, we designed a stable model of the cortico-baso-thalamocortical loops (CBG) using previously neglected anatomical data.

- After having tested this model offline, we integrated it in a simulated robot confronted to a standard survival task to assess its efficiency as an action selection mechanism. 


\subsection{Dynamic systems}

In this paper, we have investigated the stability properties of locally projected dynamical systems (IPDS) using nonlinear contraction theory. In particular, we have given a sufficient condition for a general non-autonomous (i.e. with time-varying inputs) IPDS to be globally exponentially stable. By contrast, Zhang and Nagurney (1995) only studied the stability of a fixed equilibrium point in autonomous IPDS. Thus, the novelty of our theoretical result should be noticed.

Locally projected dynamical systems have attracted great interest since they were introduced in 1993 by Dupuis and Nagurney. Indeed, this theory is central to the study of oligopolistic markets, traffic networks, commodity production, etc (Dupuis and Nagurney, 1993). As we demonstrated in this article, this theory has also proved to be a valuable tool for establishing rigorous stability properties of neural networks. In this respect, further development of the theory as well as its application to numerous problems in theoretical neuroscience may represent exciting subjects of research.

\subsection{Neuroscience}

The CBG shares a number of similarities with the previously proposed GPR model (Gurney et al., 2001b), as its selection ability relies on two off-center on-surround subcircuits. However, it includes neglected connections from the GPe to the Striatum, which provide additional selectivity. It also considers the possible role of global projections of the GPe to the STN, GPi and SNr as a regulation of the activity in the whole basal ganglia. 
We omitted two types of documented connections in the current CBG model. First, the STN projects to the GPe, GPi and SNr but also to the striatum (Parent et al., 2000). Intriguingly, the population of STN neurons projecting to the striatum does not project to the other targets, while the other STN neurons project to at least two of the other target nuclei (GPe, GPi or $\mathrm{SNr}$ ). We could not decipher the role of this striatum-projecting population and did not include it in the current model. Its unique targeting specificity suggests it could be functionally distinct from the other STN neurons. To our knowledge, no modeling study has yet proposed a functional interpretation of this connection, a question that should be explored in future works. The other missing connections concerns the fact that lateral inhibition exist in GPe and $\mathrm{SNr}$ (Park et al., 1982; Juraska et al., 1977; Deniau et al., 1982). These additional projections were added to a version of the GPR (Gurney et al., 2004a) and seemed to enhance its selectivity. We might add these connections and proceed to a similar test with the $\mathrm{CBG}$.

The GPe to striatum connections have the previously evoked functional advantage of enhancing the quality of the selection, by silencing the unselected striatal neurons. Interestingly, the striatum is known for being a relatively silent nucleus (DeLong et al., 1984), a property supposed to be induced by the specific up/down state behavior of the striatal neurons. When using simple neuron models, like leakyintegrators, it is usually difficult to reproduce this with a threshold in the transfer function only: when many channels have a strong saliences input, all the corresponding striatal neurons tend to be activated. Our model suggests that in such a case, the GPe-striatum projections may contribute to silencing the striatum.

The proposed model includes the modulatory role of the dopamine (DA) in the BG selection process only, which corresponds to the tonic level of dopaminergic input from the ventral tegmental area and the substancia nigra pars compacta (VTA and 
$\mathrm{SNc}$ ). The effects of the variation of this tonic DA level on the selection abilities of the BG has been examined in details for the GPR (Gurney et al., 2001b), and compared with symptoms of Parkinson's disease.

The role of the phasic dopamine activity in reinforcement learning, through the adaptation of the cortico-striatal synapses, is beyond the scope of our study. Nevertheless, such an extension of the CBG could allow the online adaptation of the saliences, which are here hand-tuned. The existing models of reinforcement learning in the BG are based on the temporal difference (TD) learning algorithm (Houk et al., 1995; Joel et al., 2002). These TD models are composed of two cooperating circuits: a Critic dedicated to learning to predict future reward given the current state, and an Actor, using the Critic's predictions to choose the most appropriate action. Our model can then be considered as an Actor circuit, more anatomically detailed than those usually used (simple winner-takes-all, without persistence properties). First attempts at using detailed Actor models in TD architectures for tasks requiring a single motivation have been conducted (Khamassi et al., 2004, 2005; Frank et al., 2007). Note however that the use of the current TD-learning models would not necessary be straightforward in our case: we had to use relatively complex salience computations (see appendix B), in order to solve our relatively simple task. This is caused by its multi-motivational nature, quite common in action selection problems, but which has been given only little attention in RL-related works (Dayan, 2001; Konidaris and Barto, 2006).

\subsection{Autonomous robotics}

While early action selection mechanisms were based on a purely engineer approach (Pirjanian, 1999), progress in the understanding of the physiology of the brain re- 
gions involved in action selection now allows the investigation of biomimetic action selection mechanisms. Indeed, basal ganglia models - variations of the GPR- and reticular formation models have already been used as actions selection mechanisms for autonomous robots (Montes-Gonzalez et al., 2000; Girard et al., 2003, 2005a; Humphries et al., 2005; Prescott et al., 2006).

We showed here that the CBG may exploit its cortical feedback to exhibit behavioral persistence and thus dithering avoidance, one of the fundamental properties of efficient ASMs (Tyrrell, 1993). In our experiment, this promotes energy storage and reduced energy consumption. These properties, which clearly provide a survival advantage, were also highlighted for the GPR when tested in a similar experiment (Girard et al., 2003). Thus, comparing the GPR and the CBG in exactly the same task could reveal some subtle differences which were not identified yet. Moreover, in the current version of the $\mathrm{CBG}$, these cortico-striatal feedback connections are strictly channel to channel, the possible sequence generation effects that could result from cross channel connections probably deserves additional attention.

The contraction property of the CBG also provide a fundamental advantage for an autonomous robot. It provides a theoretical certainty regarding its stability of operation, whatever the sequences of input might be. For an autonomous agent confronted to a uncontrolled environment, where all possible sequences of inputs may happen, it seems to be essential. Of course, contraction analysis does not say anything about the pertinence of the resulting stable behavior, hence the necessity of verifying the $\mathrm{CBG}$ selection properties. However, the fact that stability issues have already been evoked for previous GPR versions (Girard et al., 2005a; Prescott et al., 2006) confirms that such a rigorous proof is useful. 


\section{Acknowledgments}

B.G. and N.T. acknowledge the partial support of the European Community Neurobotics project, grant FP6-IST-001917.

\section{A If-Then-Else decision rule}

The If-Then-Else decision tree is the following:

if $E_{p}<1$ and $o n E p B l o b=$ true then

ReloadOnEp

else if $E<1$ and $E_{p}>0$ and $o n E B l o b=$ true then

ReloadOnE

else if $E<0.8$ and $E_{p}>0$ and see $E B l o b=$ true then

ApproachE

else if $E_{p}<0.8$ and see $E p B l o b=$ true then

ApproachEp

else if $E>0.7$ and $E_{p}>0.7$ then

Rest

else if $S F L<1$ or $S F R<1$ or $(S F L<1.5$ and $S F R<1.5)$ then

AvoidObstacle

else

Wander

end if 


\section{B Robot CBG saliences}

Using the sigmoid transfer function

$$
f(x)=\frac{2}{1+e^{-4 x}}-1
$$

the saliences of each action (including the frontal cortex feedback) are:

$$
\begin{gathered}
S_{R O E}=950 \times f\left(4 \times \text { onEBlob } \times E_{p} \times(1-E)\right)+0.6 \times x_{R O E}^{F C} \\
S_{R O E_{p}}=750 \times f\left(4 \times \text { onEpBlob } \times\left(1-E_{p}\right)\right)+0.2 \times x_{R O E_{p}}^{F C} \\
S_{W}=380 \\
S_{S l}=550 \times f(2 \times \max (E p \times E-0.5,0)) \\
S_{A O}=950 \times f(2 \times(\max (1.5-S F L, 0)+\max (1.5-S F R, 0)))+0.2 \times x_{A O}^{F C} \\
S_{A E}=750 \times f\left(\text { seeEBlob } \times E_{p} \times(1-E) \times(1-\text { onEBlob })\right)+0.2 \times x_{A E}^{F C} \\
S_{A E_{p}}=750 \times f\left(\text { seeEpBlob } \times\left(1-E_{p}\right) \times(1-\text { onEpBlob })\right)+0.2 \times x_{A E_{p}}^{F C}
\end{gathered}
$$

\section{References}

Alexander, G. E., Crutcher, M. D., and DeLong, M. R. (1990). Basal gangliathalamocortical circuits: Parallel substrates for motor, oculomotor, "prefrontal" and "limbic" functions. Progress in Brain Research, 85:119-146.

Alexander, G. E., DeLong, M. R., and Strick., P. L. (1986). Parallel organization of functionally segregated circuits linking basal ganglia and cortex. Annual Review of Neuroscience, 9:357-381.

Bevan, M., Booth, P., Eaton, S., and Bolam, J. (1998). Selective innervation of neostriatal interneurons by a subclass of neurons in the globus pallidus of rats. Journal of Neuroscience, 18(22):9438-9452. 
Chevalier, G. and Deniau, M. (1990). Disinhibition as a basic process of striatal functions. Trends in Neurosciences, 13:277-280.

Dayan, P. (2001). Motivated reinforcement learning. In Leen, T., Dietterich, T., and Tresp, V., editors, Neural Information Processing Systems, volume 13. The MIT Press, Cambridge, MA.

Dayan, P. and Abbott, L. (2001). Theoretical neuroscience: computational and mathematical modeling of neural systems. MIT Press.

DeLong, M., Georgopoulos, A., Crutcher, M., Mitchell, S., Richardson, R., and Alexander, G. (1984). Functional organization of the basal ganglia: contributions of single-cell recording studies. Ciba Foundation Symposium, 107:64-82.

Deniau, J.-M., Kitai, S., Donoghue, J., and Grofova, I. (1982). Neuronal interactions in the substancia nigra pars reticulata through axon collateral of the projection neurons. Experimental Brain Research, 47:105-113.

Dupuis, P. and Nagurney, A. (1993). Dynamical systems and variational inequalities. Annals of Operations Research, 44(1):7-42.

Filippov, A. (1963). Differential equations with many-valued discontinuous righthand side. Soviet Math, 4:941-945.

Frank, M., Santamaria, A., O’Reilly, R., and Willcutt, E. (2007). Testing computational models of dopamine and noradrenaline dysfunction in attention deficit/hyperactivity disorder. Neuropsychopharmacology, 32:1583-1599.

Gerkey, B., Vaughan, R., and Howard, A. (2003). The Player/Stage project: Tools for multi-robot and distributed sensor systems. In 11th International Conference on Advanced Robotics (ICAR 2003), pages 317-323, Coimbra, Portugal.

Gillies, A. and Arbruthnott, G. (2000). Computational models of the basal ganglia. Movement Disorders, 15(5):762-770.

Girard, B., Cuzin, V., Guillot, A., Gurney, K. N., and Prescott, T. J. (2003). A basal ganglia inspired model of action selection evaluated in a robotic survival task. 
Journal of Integrative Neuroscience, 2(2):179-200.

Girard, B., Filliat, D., Meyer, J.-A., Berthoz, A., and Guillot, A. (2005a). Integration of navigation and action selection in a computational model of cortico-basal ganglia-thalamo-cortical loops. Adaptive Behavior, 13(2):115-130.

Girard, B., Tabareau, N., Berthoz, A., and Slotine, J.-J. (2006). Selective amplification using a contracting model of the basal ganglia. In Alexandre, F., Boniface, Y., Bougrain, L., Girau, B., and Rougier, N., editors, NeuroComp 2006, pages $30-33$.

Girard, B., Tabareau, N., Slotine, J.-J., and Berthoz, A. (2005b). Contracting model of the basal ganglia. In Bryson, J., Prescott, T., and Seth, A., editors, Modelling Natural Action Selection: Proceedings of an International Workshop, pages 6976, Brighton, UK. AISB Press.

Gurney, K., Humphries, M., Wood, R., Prescott, T., and Redgrave, P. (2004a). Testing computational hypotheses of brain systems function: a case study with the basal ganglia. Network: Computation in Neural Systems, 15:263-290.

Gurney, K., Prescott, T., Wickens, J., and Redgrave, P. (2004b). Computational models of the basal ganglia: from membranes to robots. Trends in Neurosciences, $27: 453-459$.

Gurney, K., Prescott, T. J., and Redgrave, P. (2001a). A computational model of action selection in the basal ganglia. I. A new functional anatomy. Biological Cybernetics, 84:401-410.

Gurney, K., Prescott, T. J., and Redgrave, P. (2001b). A computational model of action selection in the basal ganglia. II. Analysis and simulation of behaviour. Biological Cybernetics, 84:411-423.

Horn, R. and Johnson, C. (1985). Matrix Analysis. Cambridge University Press.

Houk, J. C., Adams, J. L., and Barto, A. G. (1995). A model of how the basal ganglia generate and use neural signals that predict reinforcement. In Houk, 
J. C., Davis, J. L., and Beiser, D. G., editors, Models of Information Processing in the Basal Ganglia, pages 249-271. The MIT Press, Cambridge, MA.

Humphries, M., Gurney, K., and Prescott, T. (2005). Is There an Integrative Center in the Vertebrate Brain-Stem? A Robotic Evaluation of a Model of the Reticular Formation Viewed as an Action Selection Device. Adaptive Behavior, 13(2):97113.

Ioannou, P. and Sun, J. (1996). Robust Adaptive Control. Prentice Hall, Inc., Upper Saddle River, NJ, USA.

Joel, D., Niv, Y., and Ruppin, E. (2002). Actor-critic models of the basal ganglia: new anatomical and computational perspectives. Neural Networks, 15(4-6).

Juraska, J., Wilson, C., and Groves, P. (1977). The substancia nigra of the rat: a golgi study. Journal of Comparative Neurology, 172:585-600.

Khamassi, M., Girard, B., Berthoz, A., and Guillot, A. (2004). Comparing three critic models of reinforcement learning in the basal ganglia connected to a detailed actor part in a s-r task. In Groen, F., Amato, N., Bonarini, A., Yoshida, E., and Krse, B., editors, Proceedings of the Eighth International Conference on Intelligent Autonomous Systems (IAS8), pages 430-437. IOS Press, Amsterdam, The Netherlands.

Khamassi, M., Lachèze, L., Girard, B., Berthoz, A., and Guillot, A. (2005). Actorcritic models of reinforcement learning in the basal ganglia: From natural to artificial rats. Adaptive Behavior, 13(2):131-148.

Kimura, A. and Graybiel, A., editors (1995). Functions of the Cortico-Basal Ganglia Loop. Springer, Tokyo/New York.

Kita, H., Tokuno, H., and Nambu, A. (1999). Monkey globus pallidus external segment neurons projecting to the neostriatum. Neuroreport, 10(7):1476-1472. Konidaris, G. and Barto, A. (2006). An adaptive robot motivational system. In Nolfi, S., Baldassarre, G., Calabretta, R., Hallam, J., Marocco, D., Meyer, J.-A., 
Miglino, O., and Parisi, D., editors, From Animals to Animats 9: Proceedings of the 9th International Conference on the Simulation of Adaptive Behavior, volume 4095 of LNAI, pages 346-356, Berlin, Germany. Springer.

Krotopov, J. and Etlinger, S. (1999). Selection of actions in the basal ganglia thalamocortical circuits: Review and model. International Journal of Psychophysiology, 31:197-217.

Lohmiller, W. and Slotine, J. (1998). Contraction analysis for nonlinear systems. Automatica, 34(6):683-696.

Lohmiller, W. and Slotine, J. (2000). Nonlinear process control using contraction analysis. American Institute of Chemical Engineers Journal, 46(3):588-596.

Middleton, F. A. and Strick, P. L. (1994). Anatomical evidence for cerebellar and basal ganglia involvement in higher cognitive function. Science, 266:458-461.

Mink, J. W. (1996). The basal ganglia: Focused selection and inhibition of competing motor programs. Progress in Neurobiology, 50(4):381-425.

Montes-Gonzalez, F., Prescott, T. J., Gurney, K. N., Humphries, M., and Redgrave, P. (2000). An embodied model of action selection mechanisms in the vertebrate brain. In Meyer, J.-A., Berthoz, A., Floreano, D., Roitblat, H., and Wilson, S. W., editors, From Animals to Animats 6, volume 1, pages 157-166. The MIT Press, Cambridge, MA.

Parent, A., Sato, F., Wu, Y., Gauthier, J., Lévesque, M., and Parent, M. (2000). Organization of the basal ganglia: the importance of the axonal collateralization. Trends in Neuroscience, 23(10):S20-S27.

Park, M., Falls, W., and Kitai, S. (1982). An intracellular HRP study of rat globus pallidus. I. responses and light microscopic analysis. Journal of Comparative Neurology, 211:284-294.

Pirjanian, P. (1999). Behavior coordination mechanisms - state-of-the-art. Technical Report IRIS-99-375, Institute of Robotics and Intelligent Systems, School of 
Engineering, University of Southern California.

Prescott, T. J., Montes-Gonzalez, F., Gurney, K., Humphries, M. D., and Redgrave, P. (2006). A robot model of the basal ganglia: Behavior and intrinsic processing. Neural Networks, 19:31-61.

Redgrave, P., Prescott, T. J., and Gurney, K. (1999). The basal ganglia: a vertebrate solution to the selection problem? Neuroscience, 89(4):1009-1023.

Sato, F., Lavallee, P., Lévesque, M., and Parent, A. (2000). Single-axon tracing study of neurons of the external segment of the globus pallidus in primates. Journal of Comparative Neurology, 417:17-31.

Slotine, J. and Coetsee, J. (1986). Adaptive sliding controller synthesis for nonlinear systems. International Journal of Control, 43(4):1631-1651.

Slotine, J. J. E. and Lohmiller, W. (2001). Modularity, evolution, and the binding problem: a view from stability theory. Neural networks, 14(2):137-145.

Staines, W., Atmadja, S., and Fibiger, H. (1981). Demonstration of a pallidostriatal pathway by retrograde transport of HRP-labelled lectin. Brain Research, 206:446-450.

Tabareau, N. and Slotine, J. (2006). Notes on Contraction Theory. Arxiv preprint nlin.AO/0601011.

Tepper, J. and Bolam, J. (2004). Functional density and specificity of neostrital interneurons. Current Opinion in Neurobiology, 14:685-692.

Tepper, J., Koós, T., and Wilson, C. (2004). Gabaergic microcircuits in the neostriatum. Trends in Neuroscience, 11:662-669.

Tyrrell, T. (1993). The use of hierarchies for action selection. Adaptive Behavior, $1(4): 387-420$.

Wu, Y., Richard, S., and Parent, A. (2000). The organization of the striatal output system: a single-cell juxtacellular labeling study in the rat. Neuroscience Research, 38:49-62. 
Zhang, D. and Nagurney, A. (1995). On the stability of projected dynamical systems. Journal of Optimization Theory and Applications, 85(1):97-124. 


\section{List of Figures}

B.1 Cortico-baso-thalamo-cortical loops. The basal ganglia receive inputs from the whole cortex, but establish loops with the frontal areas only. Shaded arrows: inhibitory projections.

B.2 Basal ganglia model. Nuclei are represented by boxes, each circle in these nuclei represents an artificial rate-coding neuron. On this diagram, three channels are competing for selection, represented by the three neurons in each nucleus. The second channel is represented by colored shading. For clarity, the projections from the second channel neurons only are represented, they are identical for the other channels. White arrowheads represent excitations and black arrowheads, inhibitions. D1 and D2: neurons of the striatum with two respective types of dopamine receptors; STN: subthalamic nucleus; GPe: external segment of the globus pallidus; GPi/SNr: internal segment of the globus pallidus and substantia nigra pars reticulata.

B.3 Variation of the GPi/SNr inhibitory output during the Gurney et al. (2001b) test applied to (top) the CBG and (bottom) the GPR. Dashed lines represent the input salience of the channel and solid lines represent the output of the channel. Note that during the fourth step $(6 s<t<8 s)$, channels 1 and 2 are selected by the CBG, while the GPR selects channel 2 only (asterisk).

B.4 Efficiency (top) and distortion (bottom) in the winning channel for a systematic salience-space search for the CBG (left) and the GPR (right). Top: black to white gradient represents increasing efficiency (from 0 to 1 ); bottom: black to white gradient represents decreasing distortion (from 1 to 0 ), maximal distortion corresponding to simultaneous selection of both channelsis thus in black. White line: limit beyond which no selection occurs; dashed black line: diagonal representing equal saliences. For the GPR efficiency (top right), note the hysteresis area between the dashed and the full black lines. See text for further explanations.

B.5 Experimental setup. Blue square: Potential Energy resource; red square: Energy resource. The light gray surfaces represent the field of view of the sonars, and the darker one the field of view of the camera. The corresponding camera image is represented at the bottom. 
B.6 Typical dithering of the ITE between the ReloadOnEnergy and Wander actions. Top: levels of Energy (dashed line) and Potential Energy (full line); bottom: selected action. Note how during the dithering period, more than 0.3 units of $E_{p}$ are wasted in about $7 s$, while they should have allowed $30 \mathrm{~s}$ of survival.

B.7 Hysteresis in the variation of the salience of ReloadOnEnergy for the CBG. Black dashed line: variation of $S_{R O E}$ with regards to $\left(E_{p} \times(1-E)\right)$, with $o n E B l o b=1$ and without the persistence term (raw $S_{R O E}$ ); blue line: variation of $S_{R O E}$; shaded area: $S_{R O E}$ increase resulting from the frontal cortex feedback; black line: salience of Wander $\left(S_{W}\right)$. Explanations in text.

B.8 Histograms of Energy (left) and Potential Energy (right) for the CBG (top) and the ITE (bottom), cumulated over all trials.

B.9 Potential Energy consumption rate. These histograms represent the average $E_{p}$ consumption rate computed for each trial. Top: BG model; bottom: ITE; the dashed line shows the Energy consumption rate of all actions except Rest $(0.001 E / s)$. 


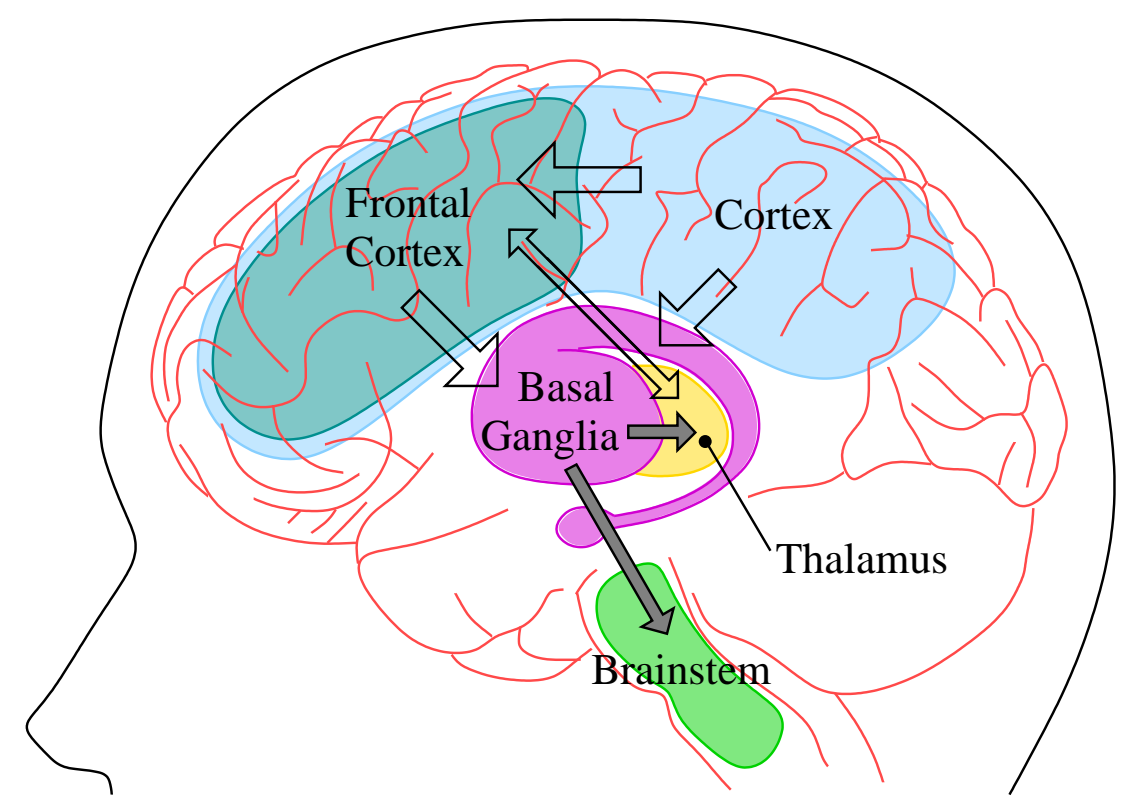

Fig. B.1. Cortico-baso-thalamo-cortical loops. The basal ganglia receive inputs from the whole cortex, but establish loops with the frontal areas only. Shaded arrows: inhibitory projections. 


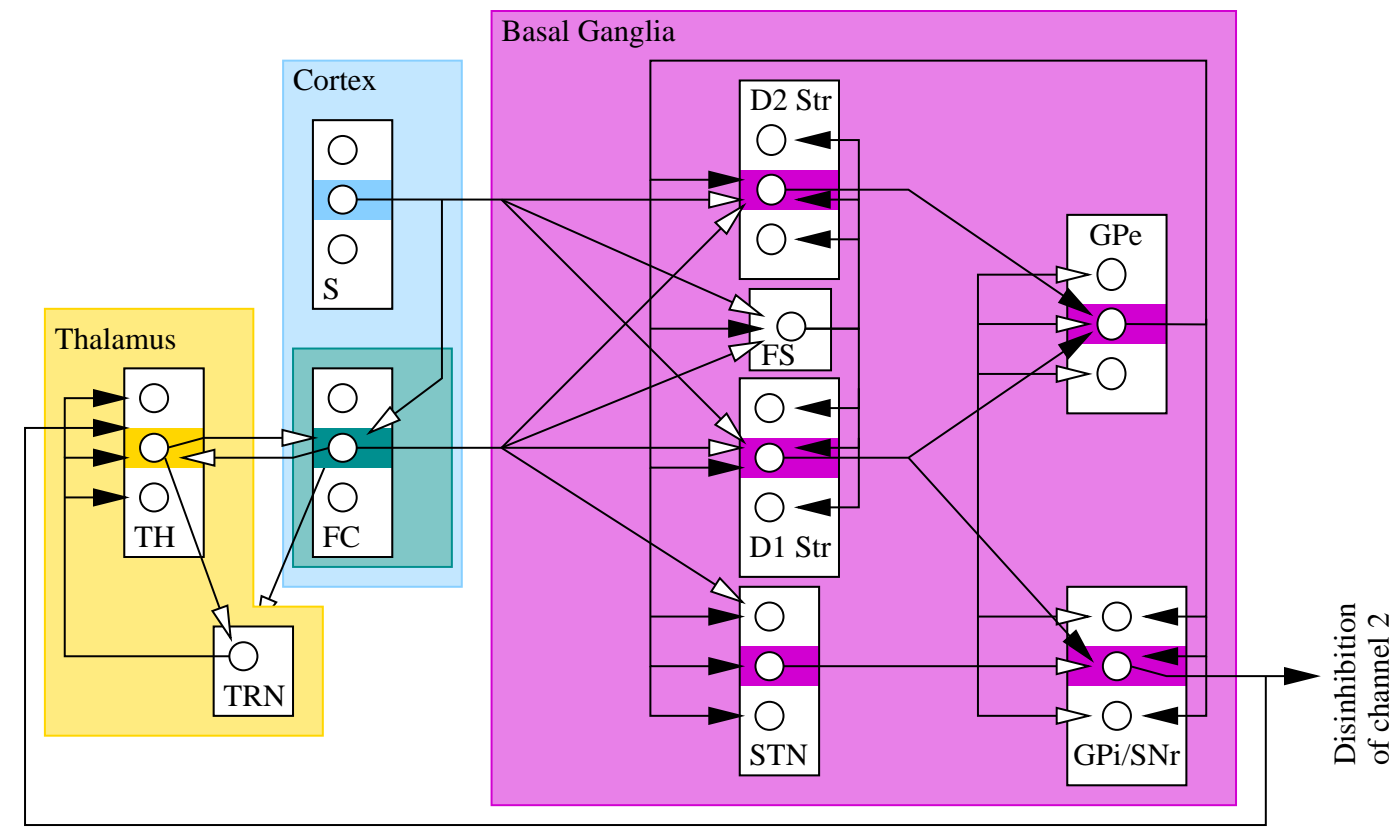

Fig. B.2. Basal ganglia model. Nuclei are represented by boxes, each circle in these nuclei represents an artificial rate-coding neuron. On this diagram, three channels are competing for selection, represented by the three neurons in each nucleus. The second channel is represented by colored shading. For clarity, the projections from the second channel neurons only are represented, they are identical for the other channels. White arrowheads represent excitations and black arrowheads, inhibitions. D1 and D2: neurons of the striatum with two respective types of dopamine receptors; STN: subthalamic nucleus; GPe: external segment of the globus pallidus; GPi/SNr: internal segment of the globus pallidus and substantia nigra pars reticulata. 

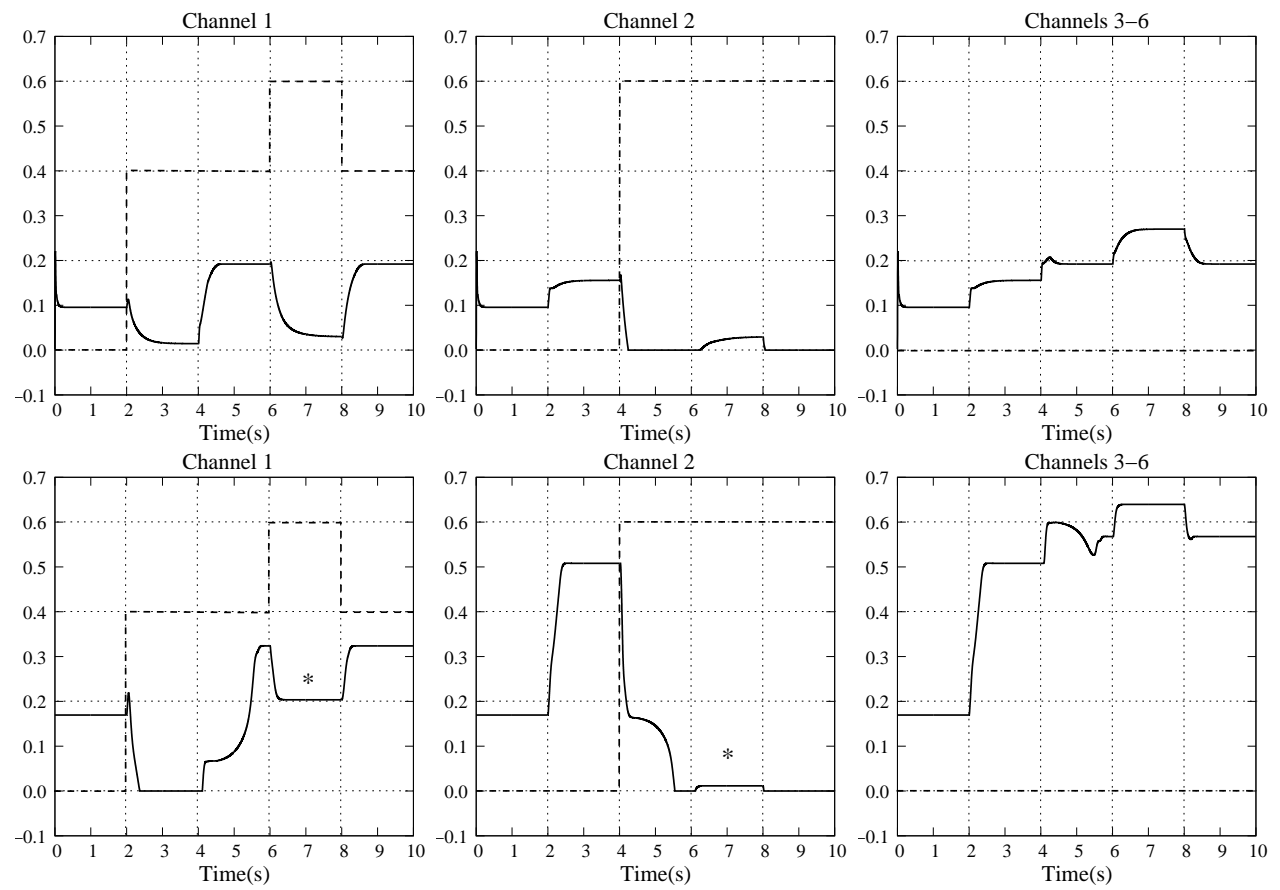

Fig. B.3. Variation of the GPi/SNr inhibitory output during the Gurney et al. (2001b) test applied to (top) the CBG and (bottom) the GPR. Dashed lines represent the input salience of the channel and solid lines represent the output of the channel. Note that during the fourth step $(6 s<t<8 s)$, channels 1 and 2 are selected by the CBG, while the GPR selects channel 2 only (asterisk). 

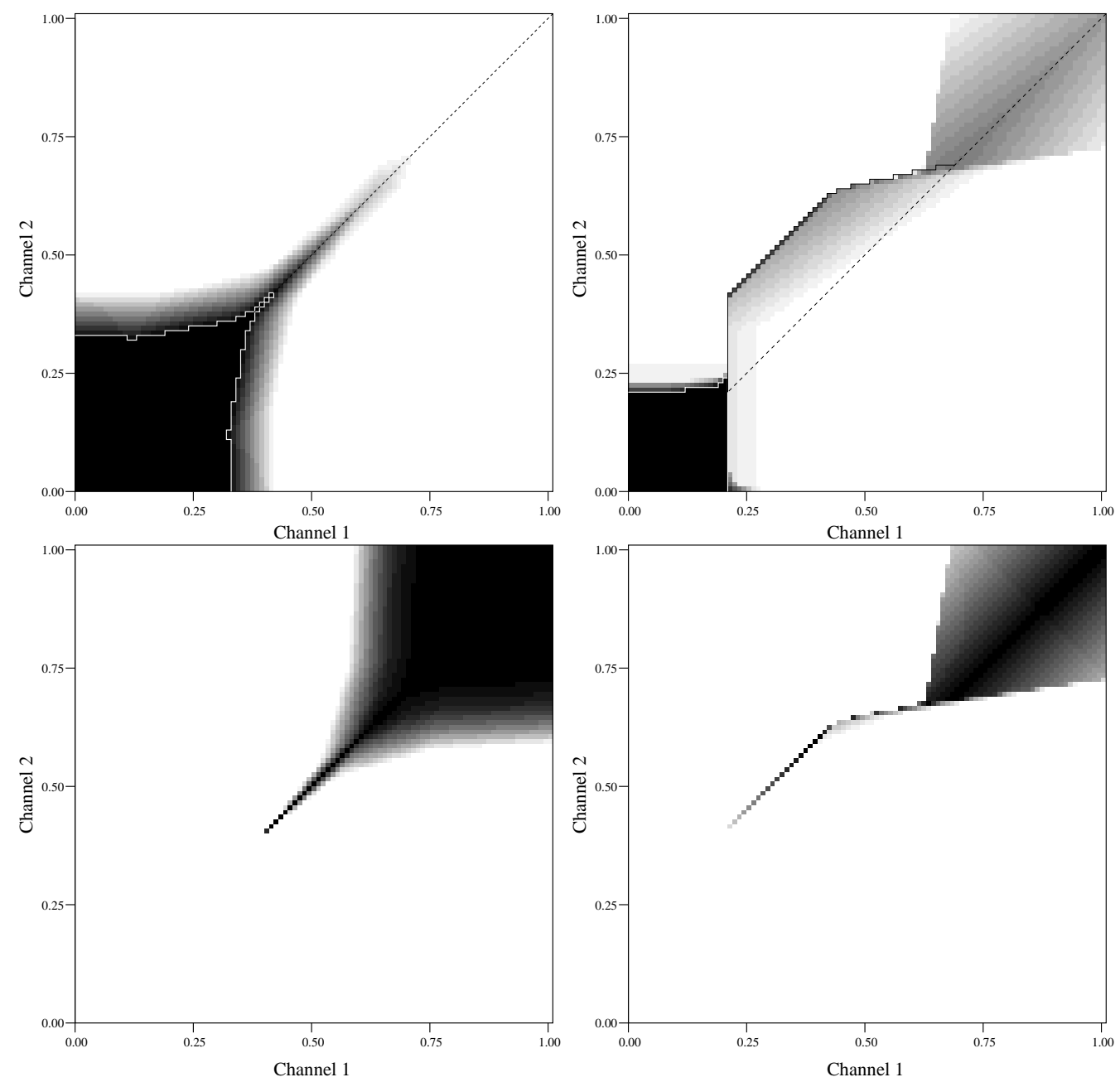

Fig. B.4. Efficiency (top) and distortion (bottom) in the winning channel for a systematic salience-space search for the CBG (left) and the GPR (right). Top: black to white gradient represents increasing efficiency (from 0 to 1 ); bottom: black to white gradient represents decreasing distortion (from 1 to 0 ), maximal distortion corresponding to simultaneous selection of both channelsis thus in black. White line: limit beyond which no selection occurs; dashed black line: diagonal representing equal saliences. For the GPR efficiency (top right), note the hysteresis area between the dashed and the full black lines. See text for further explanations. 


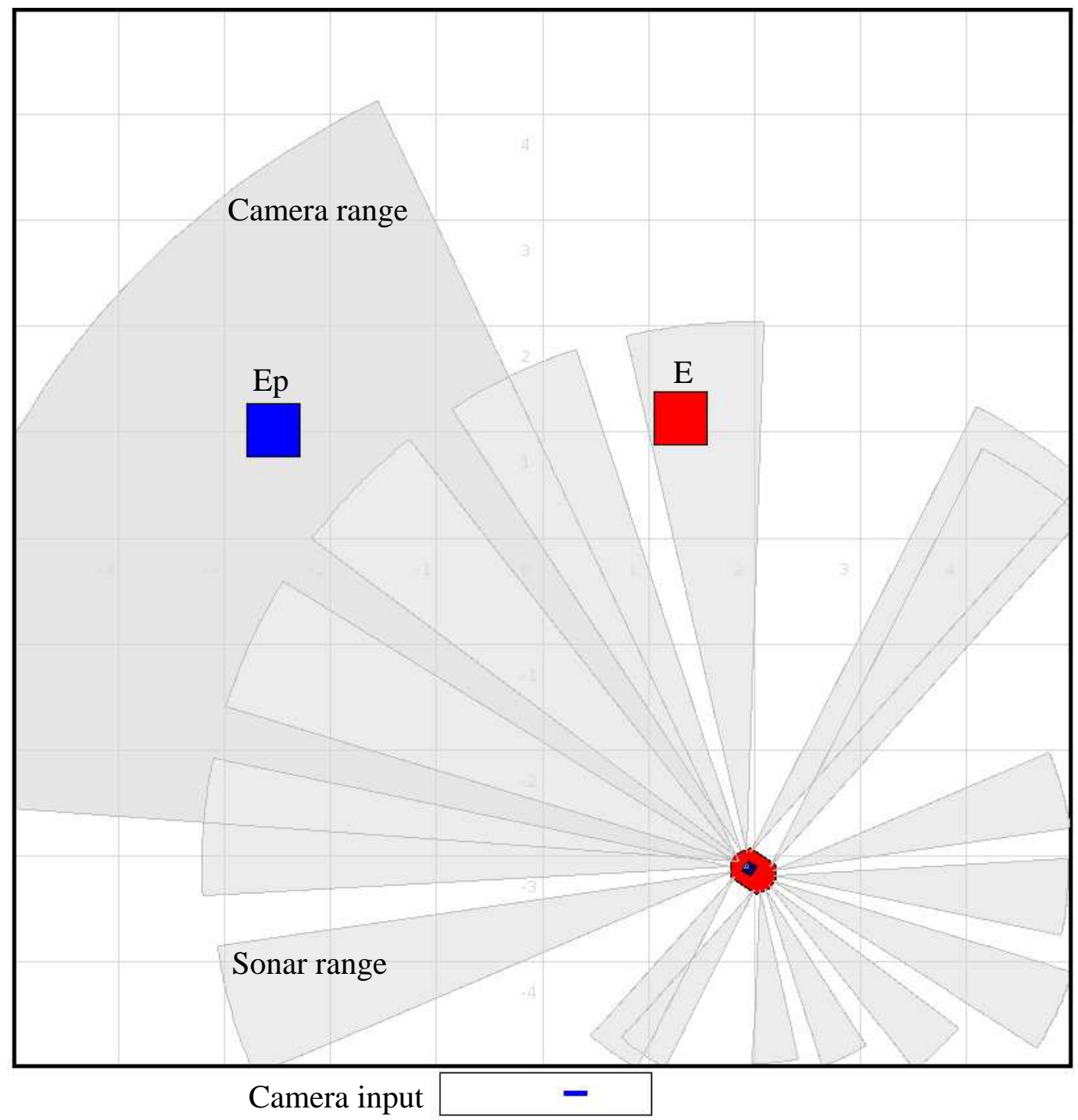

Fig. B.5. Experimental setup. Blue square: Potential Energy resource; red square: Energy resource. The light gray surfaces represent the field of view of the sonars, and the darker one the field of view of the camera. The corresponding camera image is represented at the bottom. 


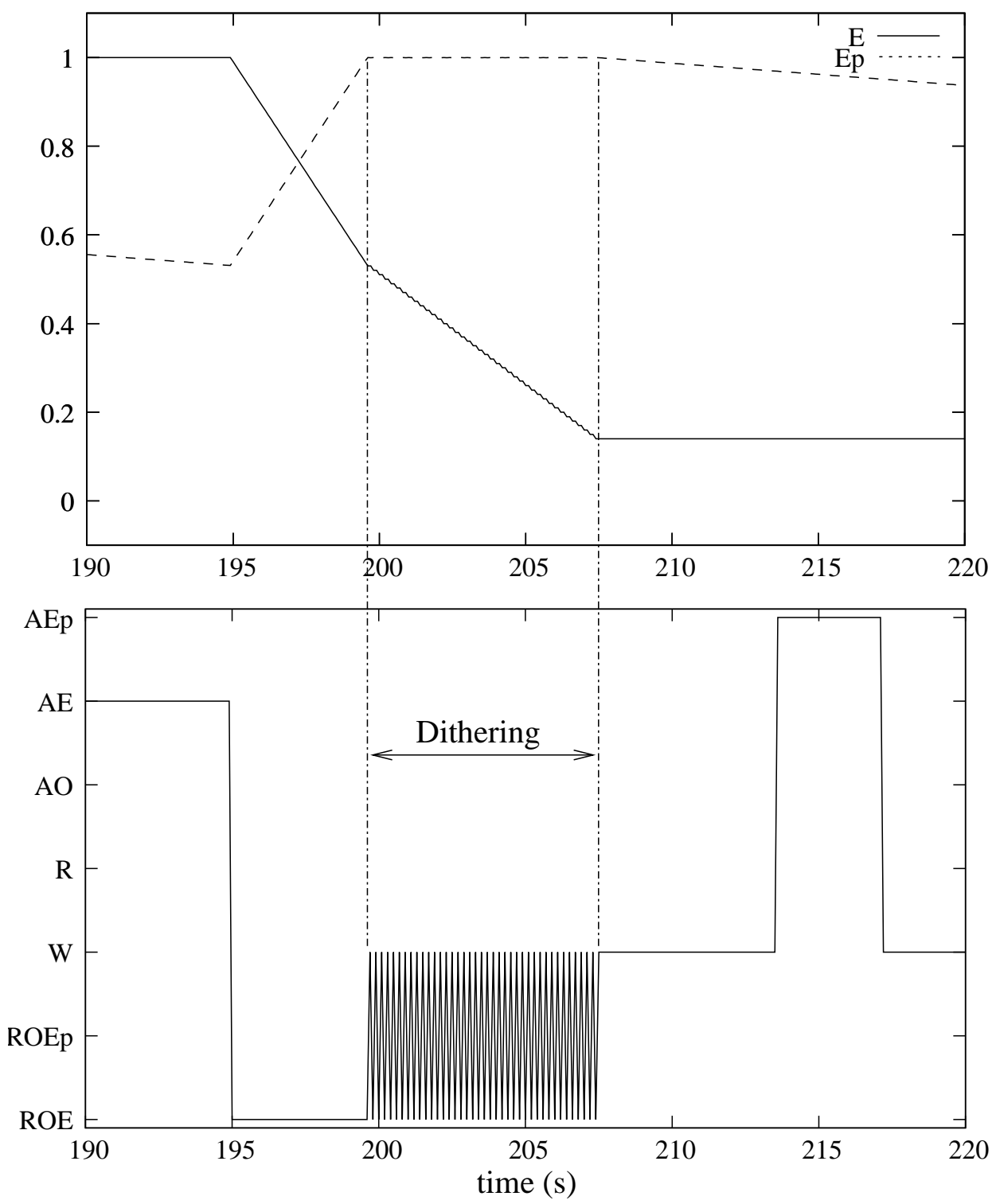

Fig. B.6. Typical dithering of the ITE between the ReloadOnEnergy and Wander actions. Top: levels of Energy (dashed line) and Potential Energy (full line); bottom: selected action. Note how during the dithering period, more than 0.3 units of $E_{p}$ are wasted in about $7 s$, while they should have allowed $30 \mathrm{~s}$ of survival. 


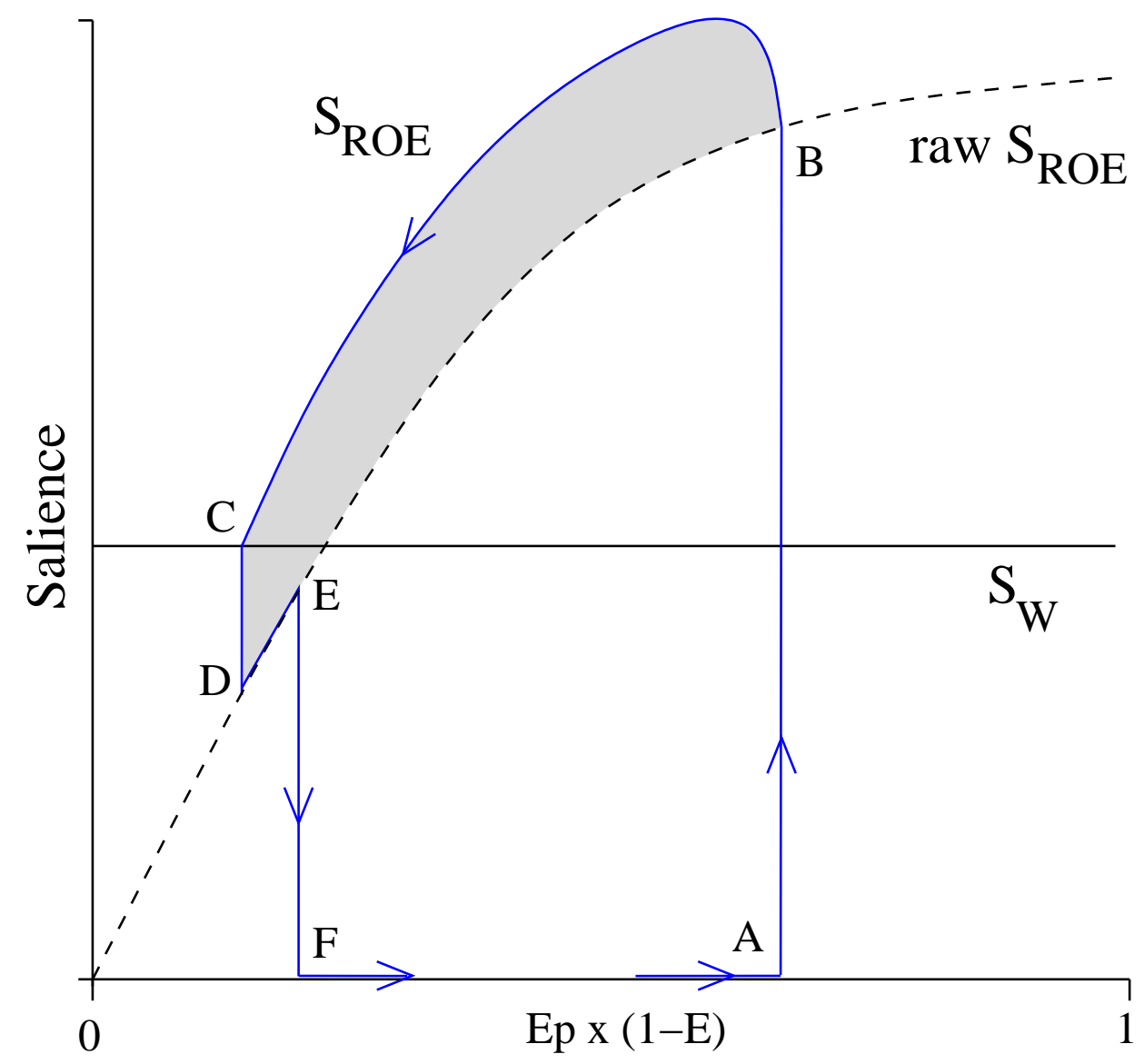

Fig. B.7. Hysteresis in the variation of the salience of ReloadOnEnergy for the CBG. Black dashed line: variation of $S_{R O E}$ with regards to $\left(E_{p} \times(1-E)\right)$, with onEBlob $=1$ and without the persistence term (raw $S_{R O E}$ ); blue line: variation of $S_{R O E}$; shaded area: $S_{R O E}$ increase resulting from the frontal cortex feedback; black line: salience of Wander $\left(S_{W}\right)$. Explanations in text. 

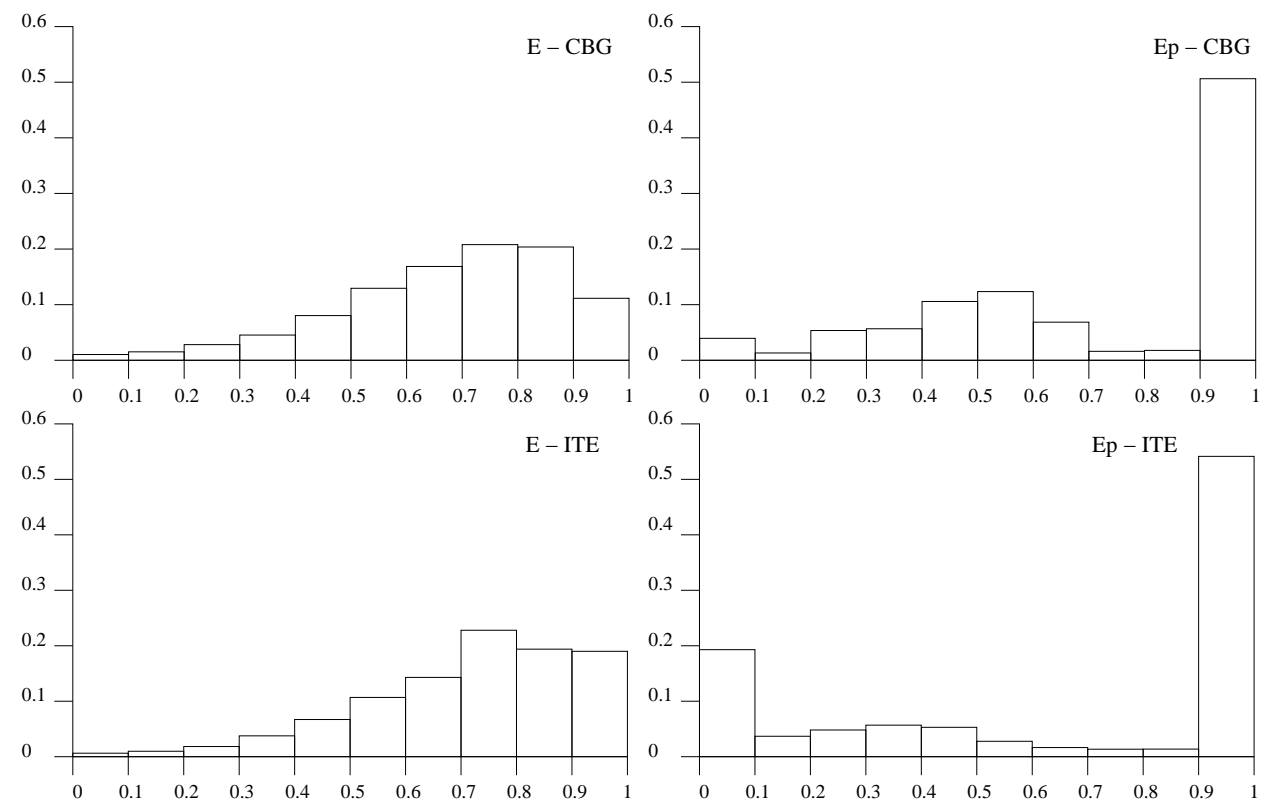

Fig. B.8. Histograms of Energy (left) and Potential Energy (right) for the CBG (top) and the ITE (bottom), cumulated over all trials. 


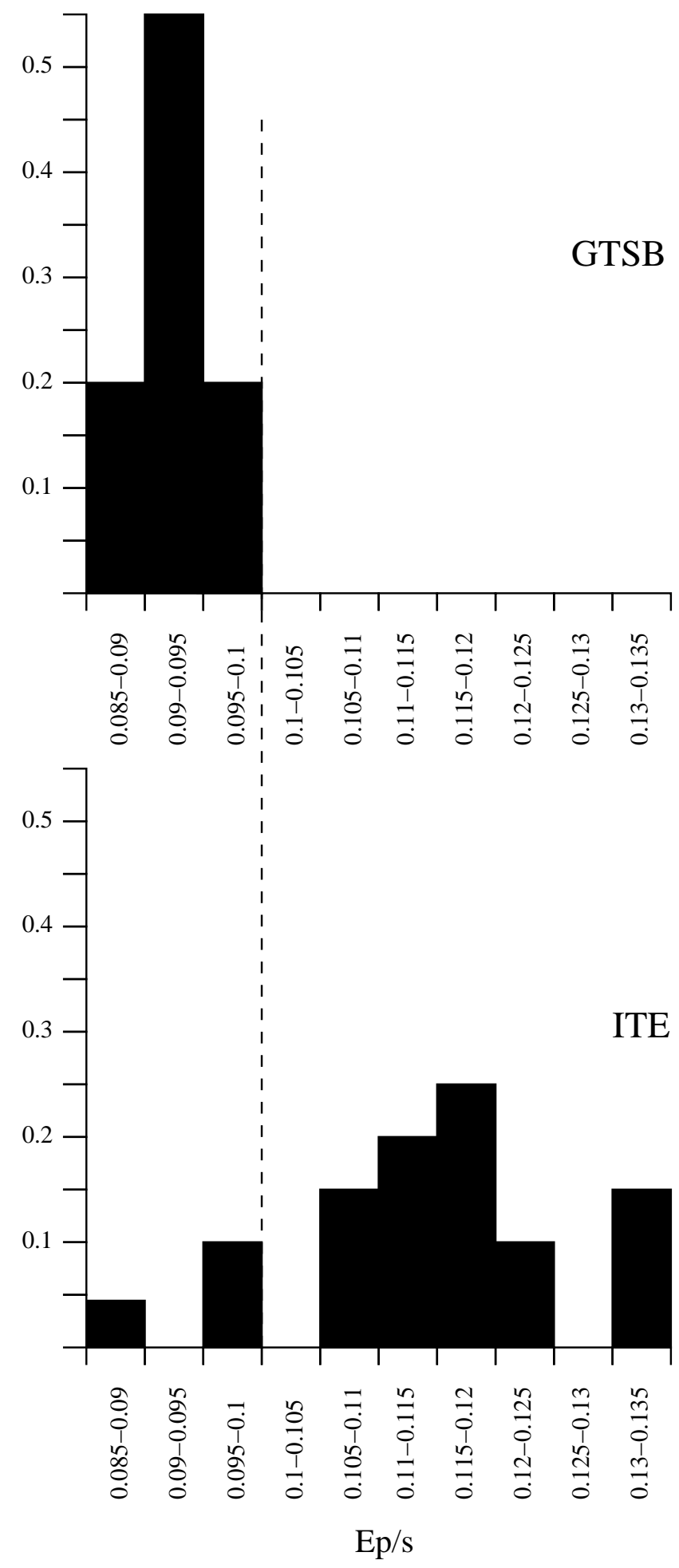

Fig. B.9. Potential Energy consumption rate. These histograms represent the average $E_{p}$ consumption rate computed for each trial. Top: BG model; bottom: ITE; the dashed line shows the Energy consumption rate of all actions except Rest $(0.001 E / s)$. 


\section{List of Tables}

B.1 Parameters of the simulations.

B.2 Value of the constants defining the metric $M_{C B G}$ for the set of parameters of our simulation 
Table B.1

Parameters of the simulations.

\begin{tabular}{c|c||c|c||c|c||c|c||c|c}
$N$ & 6 & $\tau$ & $40 m s$ & $\tau_{S T N}$ & $5 m s$ & $\tau_{F S}$ & $5 m s$ & $\tau_{F C}$ & $80 m s$ \\
\hline$\tau_{T H}$ & $5 m s$ & $\tau_{T R N}$ & $5 m s$ & $\gamma$ & 0.2 & $w_{G P e}^{D 2}$ & 1 & $w_{D 2}^{G P e}$ & 0.4 \\
\hline$w_{G P e}^{D 1}$ & 1 & $w_{D 1}^{G P e}$ & 0.4 & $w_{G P e}^{F S}$ & 0.05 & $w_{F S}^{D 1}$ & 0.5 & $w_{F S}^{D 2}$ & 0.5 \\
\hline$w_{S T N}^{G P e}$ & 0.7 & $w_{G P e}^{S T N}$ & 0.45 & $w_{G P e}^{G P i}$ & 0.08 & $w_{S T N}^{G P i}$ & 0.7 & $w_{D 1}^{G P i}$ & 0.4 \\
\hline$w_{T R N}^{T H}$ & 0.35 & $w_{T H}^{T R N}$ & 0.35 & $w_{F C}^{T H}$ & 0.6 & $w_{T H}^{F C}$ & 0.6 & $w_{F C}^{T R N}$ & 0.35 \\
\hline$w_{G P i}^{T H}$ & 0.18 & $w_{F C}^{S T N}$ & 0.58 & $w_{F C}^{D 1}$ & 0.1 & $w_{F C}^{D 2}$ & 0.1 & $w_{F C}^{F S}$ & 0.01 \\
\hline$I_{D 1}$ & -0.1 & $I_{D 2}$ & -0.1 & $I_{S T N}$ & 0.5 & $I_{G P i}$ & 0.1 & $I_{G P e}$ & 0.1
\end{tabular}


Table B.2

Value of the constants defining the metric $M_{C B G}$ for the set of parameters of our simulation

\begin{tabular}{|c|c|c|c|c|c|c|c|c|c|}
\hline$\kappa_{G P e}$ & $\kappa_{S T N}$ & $\kappa_{D 1}$ & $\kappa_{D 2}$ & $\kappa_{F S}$ & $\kappa_{G P i}$ & $\kappa_{T H}$ & $\kappa_{T R N}$ & $\kappa_{F C t x}$ & $\alpha$ \\
\hline 1 & 0.441 & 0.577 & 0.707 & 1 & 0.104 & 1 & 1 & 5.282 & 0.253 \\
\hline
\end{tabular}

